This item was submitted to Loughborough's Research Repository by the author.

Items in Figshare are protected by copyright, with all rights reserved, unless otherwise indicated.

\title{
Spin-boson model through a Poisson-driven stochastic process
}

PLEASE CITE THE PUBLISHED VERSION

http://dx.doi.org/10.1007/s00209-014-1299-1

\section{PUBLISHER}

(c) Springer Heidelberg

\section{VERSION}

AM (Accepted Manuscript)

\section{PUBLISHER STATEMENT}

This work is made available according to the conditions of the Creative Commons Attribution-NonCommercialNoDerivatives 4.0 International (CC BY-NC-ND 4.0) licence. Full details of this licence are available at: https://creativecommons.org/licenses/by-nc-nd/4.0/

\section{LICENCE}

CC BY-NC-ND 4.0

\section{REPOSITORY RECORD}

Hirokawa, Masao, Fumio Hiroshima, and Jozsef Lorinczi. 2019. "Spin-boson Model Through a Poisson-driven Stochastic Process". figshare. https://hdl.handle.net/2134/21507. 


\title{
Spin-Boson Model through a Poisson-Driven Stochastic Process
}

\author{
Masao Hirokawa \\ Department of Mathematics, Okayama University \\ Okayama, 700-8530, Japan \\ hirokawa@math.okayama-u.ac.jp \\ Fumio Hiroshima \\ Faculty of Mathematics, Kyushu University \\ Fukuoka, 819-0395, Japan \\ hiroshima@math.kyushu-u.ac.jp \\ József Lőrinczi \\ School of Mathematics, Loughborough University \\ Loughborough LE11 3TU, United Kingdom \\ J.Lorinczi@lboro.ac.uk
}

\begin{abstract}
We give a functional integral representation of the semigroup generated by the spin-boson Hamiltonian by making use of a Poisson point process and a Euclidean field. We present a method of constructing Gibbs path measures indexed by the full real line which can be applied also to more general stochastic processes with jump discontinuities. Using these tools we then show existence and uniqueness of the ground state of the spin-boson, and analyze ground state properties. In particular, we prove super-exponential decay of the number of bosons, Gaussian decay of the field operators, derive expressions for the positive integer, fractional and exponential moments of the field operator, and discuss the field fluctuations in the ground state.
\end{abstract}

Key-words: Poisson process, càdlàg paths, Gibbs measure, spin-boson operator, ground state 


\section{Spin-boson model}

\subsection{Introduction}

Gibbs measures constructed on the space of continuous paths of a random process proved to play an important role in studying ground state properties of Hamiltonians in quantum field theory ([LHB11, Chapter 6] and [LM01, LMS02, L02, BHLMS02, BH09, GL09, GHL12]). Such random processes are obtained by conditioning Brownian motion with respect to an external and a pair interaction potential. In this setting Gibbs measures are obtained as weak limits of sequences of Gibbs measures indexed by the bounded intervals of the real line by using pre-compactness or tightness arguments.

In the present paper we extend this strategy to construct Gibbs measures on paths of a random process with jump discontinuities (càdlàg paths) associated with the Hamilton operator of the spin-boson model:

$$
H=-\varepsilon \sigma_{x} \otimes \mathbb{1}+\mathbb{1} \otimes H_{\mathrm{f}}+\alpha \sigma_{z} \otimes \phi_{\mathrm{b}}(\hat{h})
$$

with a view of studying spectral properties of this Hamiltonian. Here $\varepsilon>0$ and $\alpha \in \mathbb{R}$ are parameters, $\sigma_{x}, \sigma_{z}$ are Pauli matrices, $H_{\mathrm{f}}$ is the free field Hamiltonian, $\phi_{\mathrm{b}}(\hat{h})$ is the field operator in Fock space $\mathscr{F}$, and $\hat{h}$ is a form factor (see the details below). One of the merits of this approach is that it allows to carry through this analysis in a nonperturbative way. While in [Spo89, SSW90] the spectral properties of the spin-boson model are discussed through a measure on the space of paths with jump discontinuities, no attention was paid to constructing Gibbs measures.

As it will be seen below, in the case of the spin-boson model Gibbs measures involve densities dependent on a pair interaction potential alone, and no external potential contribution. We stress that, in contrast, in the case of Brownian motion under zero external potential and non-zero pair interaction potential even the very existence of Gibbs measures is poorly understood. A rigorous study of Gibbs measures with an external potential but without pair interaction on càdlàg paths was begun in [KL12a], considering them with respect to fractional $P(\phi)_{1}$-processes, i.e., stable processes under an external potential.

For Hamiltonians with spin or a non-local kinetic term Feynman-Kac-type formulae can be derived by using Lévy processes [Hir12, HL08, LM12, HIL12a, HIL12b, KL12b]. There are few rigorous results on quantum models with spin using functional integration methods. In [HL08] we derived such a formula for the heat semigroup generated by a quantum field operator with spin by making use of a Euclidean quantum field and a 
Poisson process. In [HIL12b] we developed similar methods for relativistic Schrödinger operators, allowing to obtain results on the decay of ground states.

In this paper we derive a Feynman-Kac-type formula for the semigroup generated by the spin-boson Hamiltonian. The spin-boson model is a much studied variant of the Caldeira-Leggett model describing a two-state quantum system linearly coupled to a scalar quantum field [LCDGZ87]. Work on the spectral properties of the spinboson and related models includes [SD85, Spo89, SSW90, HS95, Ger96, BS98, AH97, Hir99, Ger00, Hir01, Hir02]. In particular, in [SD85, Spo89, SSW90, HS95] stochastic methods were used. Existence or absence of a spin-boson ground state was investigated in [AH97, Ger00]. Below we obtain existence and uniqueness of the ground state by a different approach. In [HH12] we apply the method developed in this paper to the so-called Rabi model, which can be regarded as a single-mode spin-boson model. We also refer to the recent papers [Abd12, HH10].

In order to study the spin-boson in a stochastic representation we describe the spin states by the set $\mathbb{Z}_{2}=\{-1,+1\}$ and derive a Poisson-driven random process with càdlàg path space $\mathscr{X}=D\left(\mathbb{R}, \mathbb{Z}_{2}\right)$, indexed by the real line and taking values in $\mathbb{Z}_{2}$. This will describe the spin-process. The spin-boson Hamiltonian can be defined as a self-adjoint operator on a Hilbert space $L^{2}\left(\mathbb{Z}_{2} \times Q\right)$ instead of $\mathbb{C}^{2} \otimes \mathscr{F}$.

On path space $\mathscr{X}$ we are then able to construct a Gibbs measure $\mu_{\mathrm{g}}$ associated with the unique ground state $\varphi_{\mathrm{g}}$ of the spin-boson Hamiltonian. Using this probability measure we represent ground state expectations for interesting choices of operators $\mathcal{O}$ in the form

$$
\left(\varphi_{\mathrm{g}}, \mathcal{O} \varphi_{\mathrm{g}}\right)=\int_{\mathscr{X}} f_{\mathcal{O}} d \mu_{\mathrm{g}}
$$

where $f_{\mathcal{O}}$ is a function on path space $\mathscr{X}$ uniquely associated with $\mathcal{O}$. We will consider the field operator $\phi(f)$ with test function $f$, and the second quantization $d \Gamma(\rho(-i \nabla))$ of the multiplication operator by a function $\rho$, and derive path integral representations of expressions of the type

$$
\mathcal{O}=\xi(\sigma) F(\phi(f)) \quad \text { and } \quad \mathcal{O}=\xi(\sigma) e^{-\beta d \Gamma(\rho(-i \nabla))}
$$

with suitable $\xi: \mathbb{Z}_{2} \rightarrow \mathbb{C}, F: \mathbb{R} \rightarrow \mathbb{C}$ and $\rho: \mathbb{R}^{d} \rightarrow[0, \infty)$. These cases include

$$
\mathcal{O}=N^{m}, \quad \mathcal{O}=e^{\beta N}, \quad \mathcal{O}=(-1)^{N} \quad \text { and } \quad \sigma(-1)^{N}
$$

for all $m \in \mathbb{N}$ and $\beta \in \mathbb{C}$ (in particular, $\beta>0$ ), where $N=d \Gamma(\mathbb{1})$ is the boson number operator, as well as

$$
\mathcal{O}=e^{(\beta / 2) \phi(f)^{2}}, \quad \mathcal{O}=e^{i \beta \phi(f)}, \quad \mathcal{O}=\phi(f)^{n}, \quad \mathcal{O}=|\phi(f)|^{s} \quad \text { and } \quad \mathcal{O}=\sigma \phi(f) .
$$


Here $\beta \in \mathbb{R}, n \in \mathbb{N}$ and $0<s<2$.

Specifically, we obtain explicit formulae for the positive integer and fractional order moments and exponential moments of the field operator, and show that the field fluctuations increase on switching on the coupling between the spin and the boson field. Moreover, we show that

$$
\varphi_{\mathrm{g}} \in D\left(e^{(\beta / 2) \phi(f)^{2}}\right)
$$

for $-\infty<\beta<1 /\|f\|^{2}$ (i.e., Gaussian decay of the field operators) with

$$
\lim _{\beta \uparrow 1 /\|f\|^{2}}\left\|e^{(\beta / 2) \phi(f)^{2}} \varphi_{\mathrm{g}}\right\|=\infty .
$$

As a consequence, we obtain another representation of the ground state. Recall that when $\varepsilon=0$, the spin boson Hamiltonian can be diagonalized so that each matrix element is a van Hove Hamiltonian (see also below). Then it is trivial to see that

$$
\left(\varphi_{\mathrm{g}}, F(\phi(f)) \varphi_{\mathrm{g}}\right)=\left(\varphi_{\mathrm{vH}}, F\left(\phi_{\mathrm{b}}(\hat{f})\right) \varphi_{\mathrm{vH}}\right)
$$

where $\varphi_{\mathrm{vH}}$ is the ground state of the van Hove Hamiltonian in $\mathscr{F}$. Here we show a similar representation for the case of $\varepsilon \neq 0$, i.e., we derive

$$
\left(\varphi_{\mathrm{g}}, F(\phi(f)) \varphi_{\mathrm{g}}\right)=\int_{\mathscr{X}}\left(\varphi_{\mathrm{vH}}(\chi), F\left(\phi_{\mathrm{b}}(\hat{f})\right) \varphi_{\mathrm{vH}}(\chi)\right) d \mu_{\mathrm{g}}
$$

where $\varphi_{\mathrm{vH}}(\chi)$ is the ground state of a random van Hove model and $\chi$ is a function of the random path. This suggests implicitly that $\varphi_{\mathrm{g}}=\int_{\mathscr{X}}^{\oplus} \varphi_{\mathrm{vH}}(\chi) d \mu_{\mathrm{g}}$.

Next we discuss ground state properties of second quantized operators. In particular, we prove that

$$
\varphi_{\mathrm{g}} \in D\left(e^{\beta N}\right), \quad \beta>0
$$

(i.e., super-exponential decay of the number of bosons). Also, we obtain explicit formulae for the moments of the boson number operator in terms of sums involving coefficients given by the Stirling numbers of the second kind. Furthermore, although we show that $\left(\varphi_{\mathrm{g}}, \sigma(-1)^{N} \varphi_{\mathrm{g}}\right)=-1$, we obtain a positive lower bound on the ground state functional $\left(\varphi_{\mathrm{g}},(-1)^{N} \varphi_{\mathrm{g}}\right)$. Finally, we obtain the inequality

$$
\left(\varphi_{\mathrm{g}}, N \varphi_{\mathrm{g}}\right) \leq \frac{\alpha}{2}\left(\varphi_{\mathrm{g}}, \phi\left(\omega(\mathrm{D})^{-1} h\right) \varphi_{\mathrm{g}}\right) \leq \frac{\alpha^{2}}{2}\|\hat{h} / \omega\|^{2}, \quad \mathrm{D}=-i \nabla,
$$

relating the mean of the field operator with the expected boson number in the ground state.

These applications to ground state properties are derived from the main results of this paper, which can be summarized as follows: 
(1) existence and uniqueness of the ground state $\varphi_{\mathrm{g}}$ of the spin-boson Hamiltonian $H$ is shown in Theorem 2.3

(2) a probability measure $\mu_{\mathrm{g}}$ on càdlàg path space $\mathscr{X}$ associated with $\varphi_{\mathrm{g}}$ is constructed in Theorems 3.8 and 3.9

(3) it is shown in Theorem 3.12 that $\mu_{\mathrm{g}}$ is a Gibbs measure for a pair interaction potential

(4) path integral representations of $\left(\varphi_{\mathrm{g}}, \mathcal{O} \varphi_{\mathrm{g}}\right)$ in terms of $\mu_{\mathrm{g}}$ are given in Section 4, in particular,

(i) it is shown that

$$
\varphi_{\mathrm{g}} \in D\left(e^{a N}\right) \cap D\left(e^{b \phi(f)^{2}}\right), \quad(a, b) \in \mathbb{R} \times\left(-\infty, 1 /\|f\|^{2}\right)
$$

in Theorem 4.9, Corollary 4.10 and Corollary 4.18

(ii) it is shown that $\left(\varphi_{\mathrm{g}}, \mathcal{O} \varphi_{\mathrm{g}}\right)$ can be represented by the ground state $\varphi_{\mathrm{vH}}(\chi)$ of a van Hove Hamiltonian as

$$
\left(\varphi_{\mathrm{g}}, \mathcal{O} \varphi_{\mathrm{g}}\right)=\int_{\mathscr{X}}\left(\varphi_{\mathrm{vH}}(\chi), \mathcal{O} \varphi_{\mathrm{vH}}(\chi)\right) d \mu_{\mathrm{g}}
$$

in Theorem 4.14.

We note that path integral representations were already used to a great extent for the Nelson model describing the interaction of a charged particle with a scalar quantum field, see [BHLMS02] and [LHB11, Chapter 6]. We also note that although the result $\left(\varphi_{\mathrm{g}}, e^{\beta N} \varphi_{\mathrm{g}}\right)<\infty$ has been established in [Gro73] by using operator theory, our construction here is completely different and rather general. Also, our methods can be applied to further models involving càdlàg paths, for instance, the Nelson model with a relativistic kinetic term $\sqrt{-\Delta+m^{2}}+V$, which will be done elsewhere.

The paper is organized as follows. The remainder of Section 1 is devoted to constructing the Feynman-Kac formula of the spin-boson heat semigroup. In Section 2 we show that the spin-boson Hamiltonian has a unique ground state $\varphi_{\mathrm{g}}$ if an infrared regularity condition is satisfied. In Section 3 we define a Gibbs measure on $\mathscr{X}$ for bounded time intervals associated with the density obtained from the Feynman-Kac representation, and show its local weak convergence to a path measure in the infinite time limit. We view Theorems 3.8 and 3.9 below to be pivotal results in this paper. In Section 4 we derive the expressions of the ground state expectations mentioned above. 


\subsection{Definition}

We begin by defining the spin-boson Hamiltonian as a self-adjoint operator on a Hilbert space. Let $\mathscr{F}=\bigoplus_{n=0}^{\infty}\left(\otimes_{\text {sym }}^{n} L^{2}\left(\mathbb{R}^{d}\right)\right)$ be the boson Fock space over $L^{2}\left(\mathbb{R}^{d}\right)$, where the subscript means symmetrized tensor product. We denote the boson annihilation and creation operators by $a(f)$ and $a^{\dagger}(f), f, g \in L^{2}\left(\mathbb{R}^{d}\right)$, respectively, satisfying the canonical commutation relations

$$
\left[a(f), a^{\dagger}(g)\right]=(\bar{f}, g), \quad[a(f), a(g)]=0=\left[a^{\dagger}(f), a^{\dagger}(g)\right] .
$$

We use the informal expression $a^{\sharp}(f)=\int a^{\sharp}(k) f(k) d k$ for notational convenience. Consider the Hilbert space

$$
\mathscr{H}=\mathbb{C}^{2} \otimes \mathscr{F} .
$$

Denote by $d \Gamma(T)$ be the second quantization of a self-adjoint operator $T$ in $L^{2}\left(\mathbb{R}^{d}\right)$. The operator on Fock space defined by

$$
H_{\mathrm{f}}=d \Gamma(\omega)
$$

is the free boson Hamiltonian with dispersion relation $\omega(k)=|k|$. The operator

$$
\phi_{\mathrm{b}}(\hat{h})=\frac{1}{\sqrt{2}} \int\left(a^{\dagger}(k) \hat{h}(-k)+a(k) \hat{h}(k)\right) d k,
$$

acting on Fock space is the scalar field operator, where $h \in L^{2}\left(\mathbb{R}^{d}\right)$ is a suitable form factor and $\hat{h}$ is the Fourier transform of $h$. Denote by $\sigma_{x}, \sigma_{y}$ and $\sigma_{z}$ the $2 \times 2$ Pauli matrices given by

$$
\sigma_{x}=\left[\begin{array}{ll}
0 & 1 \\
1 & 0
\end{array}\right], \quad \sigma_{y}=\left[\begin{array}{cc}
0 & -i \\
i & 0
\end{array}\right], \quad \sigma_{z}=\left[\begin{array}{cc}
1 & 0 \\
0 & -1
\end{array}\right]
$$

With these components, the spin-boson Hamiltonian is defined by the linear operator

$$
H_{\mathrm{SB}}=\varepsilon \sigma_{z} \otimes \mathbb{1}+\mathbb{1} \otimes H_{\mathrm{f}}+\alpha \sigma_{x} \otimes \phi_{\mathrm{b}}(\hat{h})
$$

on $\mathscr{H}$, where $\alpha \in \mathbb{R}$ is a coupling constant and $\varepsilon \geq 0$ a parameter.

\subsection{A Feynman-Kac-type formula}

In this section we give a functional integral representation of $e^{-t H_{\mathrm{SB}}}$ by making use of a Poisson point process and an infinite dimensional Ornstein-Uhlenbeck process. First we transform $H_{\mathrm{SB}}$ in a convenient form to study its spectrum in terms of path measures. 
Recall that the rotation group in $\mathbb{R}^{3}$ has an adjoint representation on $S U(2)$. Let $n \in$ $\mathbb{R}^{3}$ be a unit vector and $\theta \in[0,2 \pi)$. Thus $e^{(i / 2) \theta n \cdot \sigma}$ satisfies that $e^{(i / 2) \theta n \cdot \sigma} \sigma_{\mu} e^{-(i / 2) \theta n \cdot \sigma}=$ $(R \sigma)_{\mu}$, where $R$ denotes the $3 \times 3$ matrix representing the rotation around $n$ with angle $\theta$, and $\sigma=\left(\sigma_{x}, \sigma_{y}, \sigma_{z}\right)$. In particular, for $n=(0,1,0)$ and $\theta=\pi / 2$, we have

$$
\begin{aligned}
& e^{(i / 2) \theta n \cdot \sigma} \sigma_{x} e^{-(i / 2) \theta n \cdot \sigma}=\sigma_{z}, \\
& e^{(i / 2) \theta n \cdot \sigma} \sigma_{z} e^{-(i / 2) \theta n \cdot \sigma}=-\sigma_{x} .
\end{aligned}
$$

Let

$$
U=\exp \left(i \frac{\pi}{4} \sigma_{y}\right) \otimes \mathbb{1}=\frac{1}{\sqrt{2}}\left[\begin{array}{cc}
1 & 1 \\
-1 & 1
\end{array}\right] \otimes \mathbb{1}
$$

be a unitary operator on $\mathscr{H}$. By (1.10) and (1.11) $H_{\mathrm{SB}}$ transforms as

$$
H=U H_{\mathrm{SB}} U^{*}=-\varepsilon \sigma_{x} \otimes \mathbb{1}+\mathbb{1} \otimes H_{\mathrm{f}}+\alpha \sigma_{z} \otimes \phi_{\mathrm{b}}(\hat{h}) .
$$

Then $H$ is realized as

$$
H=\left[\begin{array}{cc}
H_{\mathrm{f}}+\alpha \phi_{\mathrm{b}}(\hat{h}) & -\varepsilon \\
-\varepsilon & H_{\mathrm{f}}-\alpha \phi_{\mathrm{b}}(\hat{h})
\end{array}\right]
$$

In particular, $\varepsilon=0$ makes $H$ diagonal. If $\hat{h} / \sqrt{\omega} \in L^{2}\left(\mathbb{R}^{d}\right)$ and $h$ is real-valued, then $\phi_{\mathrm{b}}(\hat{h})$ is symmetric and infinitesimally small with respect to $H_{\mathrm{f}}$, hence by the KatoRellich theorem it follows that $H$ is a self-adjoint operator on $D\left(H_{\mathrm{f}}\right)$ and bounded from below.

To construct the functional integral representation of the semigroup $e^{-t H}$, it is useful to introduce a spin variable $\sigma \in \mathbb{Z}_{2}$, where $\mathbb{Z}_{2}=\{-1,+1\}$ is the additive group of order 2. For $\Psi=\left[\begin{array}{l}\Psi(+) \\ \Psi(-)\end{array}\right] \in \mathscr{H}$, we have

$$
H \Psi=\left[\begin{array}{l}
\left(H_{\mathrm{f}}+\alpha \phi_{\mathrm{b}}(\hat{h})\right) \Psi(+)-\varepsilon \Psi(-) \\
\left(H_{\mathrm{f}}-\alpha \phi_{\mathrm{b}}(\hat{h})\right) \Psi(-)-\varepsilon \Psi(+)
\end{array}\right]
$$

Thus we can transform $H$ on $\mathscr{H}$ to the operator $\tilde{H}$ on

$$
L^{2}\left(\mathbb{Z}_{2} ; \mathscr{F}\right)=\left\{f: \mathbb{Z}_{2} \rightarrow \mathscr{F} \mid\|f\|_{L^{2}\left(\mathbb{Z}_{2} ; \mathscr{F}\right)}=\sum_{\sigma \in \mathbb{Z}_{2}}\|f(\sigma)\|_{\mathscr{F}}^{2}<\infty\right\}
$$

by

$$
(\tilde{H} \Psi)(\sigma)=\left(H_{\mathrm{f}}+\alpha \sigma \phi_{\mathrm{b}}(\hat{h})\right) \Psi(\sigma)+\varepsilon \Psi(-\sigma), \quad \sigma \in \mathbb{Z}_{2}
$$


In what follows, we identify the Hilbert space $\mathscr{H}$ with $L^{2}\left(\mathbb{Z}_{2} ; \mathscr{F}\right)$ through

$$
\mathscr{H} \ni\left[\begin{array}{l}
\Psi(+) \\
\Psi(-)
\end{array}\right] \mapsto \Psi(\sigma)=\left\{\begin{array}{ll}
\Psi(+), & \sigma=+1, \\
\Psi(-), & \sigma=-1
\end{array} \in L^{2}\left(\mathbb{Z}_{2} ; \mathscr{F}\right)\right.
$$

and instead of $H$ we consider $\tilde{H}$, and use the notation $H$ for $\tilde{H}$.

Let $(\Omega, \Sigma, P)$ be a probability space, and $\left(N_{t}\right)_{t \in \mathbb{R}}$ be a two-sided Poisson process with unit intensity on this space. We denote by $D=\left\{t \in \mathbb{R} \mid N_{t+} \neq N_{t-}\right\}$ the set of jump points, and define the integral with respect to this Poisson process by

$$
\int_{(s, t]} f\left(r, N_{r}\right) d N_{r}=\sum_{\substack{r \in D \\ r \in(s, t]}} f\left(r, N_{r}\right)
$$

for any predictable function $f$ (we refer to Appendix of [HL08] for details). In particular, we have for any continuous function $g$,

$$
\int_{(s, t]} g\left(r, N_{r-}\right) d N_{r}=\sum_{\substack{r \in D \\ s<r \leq t}} g\left(r, N_{r-}\right) .
$$

We write $\int_{s}^{t+} \cdots d N_{r}$ for $\int_{(s, t]} \cdots d N_{r}$. Note that $\int_{s}^{t+} g\left(r, N_{-r}\right) d N_{r}$ is right-continuous in $t$ and the integrand $g\left(r, N_{-r}\right)$ is left-continuous in $r$ and thus a predictable process. Define the random process

$$
\sigma_{t}=\sigma(-1)^{N_{t}}, \quad \sigma \in \mathbb{Z}_{2}
$$

This process describes the spin. Since our Poisson process is indexed by the real line, we summarize its properties below.

Proposition 1.1 The stochastic process $\left(N_{t}\right)_{t \in \mathbb{R}}$ has the following properties:

1. Independence: The random variables $N_{t}$ and $N_{s}$ are independent for all $s \leq 0 \leq t$, $s \neq t$.

2. Markov property: The stochastic processes $\left(N_{t}\right)_{t \geq 0}$ and $\left(N_{t}\right)_{t \leq 0}$ are Markov processes with respect to the natural filtrations $\mathscr{N}_{t}^{+}=\sigma\left(N_{s}, 0 \leq s \leq t\right)$ and $\mathscr{N}_{t}^{-}=\sigma\left(N_{s}, t \leq s \leq 0\right)$, respectively, i.e.,

$$
\mathbb{E}_{P}\left[N_{t+s} \mid \mathscr{N}_{s}^{+}\right]=\mathbb{E}_{P}^{N_{s}}\left[N_{t}\right], \quad \mathbb{E}_{P}\left[N_{-t-s} \mid \mathscr{N}_{-s}^{-}\right]=\mathbb{E}_{P}^{N_{-s}}\left[N_{-t}\right]
$$

3. Reflection symmetry: The random variables $N_{t}$ and $N_{-t}$ are identically distributed for all $t \in \mathbb{R}$, i.e., $\mathbb{E}_{P}\left[f\left(N_{-t}\right)\right]=\mathbb{E}_{P}\left[f\left(N_{t}\right)\right]=\sum_{n=0}^{\infty} f(n) \frac{|t|^{n}}{n !} e^{-|t|}$. 
4. Shift invariance: The stochastic process $\sigma_{t}=\sigma(-1)^{N_{t}}, t \in \mathbb{R}$, is shift invariant, i.e.,

$$
\sum_{\sigma \in \mathbb{Z}_{2}} \mathbb{E}_{P}\left[\prod_{j=0}^{n} f_{j}\left(\sigma_{t_{j}}\right)\right]=\sum_{\sigma \in \mathbb{Z}_{2}} \mathbb{E}_{P}\left[\prod_{j=0}^{n} f_{j}\left(\sigma_{s+t_{j}}\right)\right], \quad s \in \mathbb{R} .
$$

Proof: The proof is a minor modification of [LHB11, Theorem 3.106] and it is omitted.

In the Schrödinger representation the boson Fock space $\mathscr{F}$ can be realized as an $L^{2}$-space over a probability space $(Q, \mu)$, and the field operator $\phi_{\mathrm{b}}(\hat{f})$ with real-valued function $f \in L^{2}\left(\mathbb{R}^{d}\right)$ as a multiplication operator, which we will denote by $\phi(f)$. The identity function $\mathbb{1}$ on $Q$ corresponds to the Fock vacuum $\Omega_{\mathrm{b}}$ in $\mathscr{F}$.

Next we introduce the random process describing the free boson field $H_{\mathrm{f}}$. Let $\left(Q_{\mathrm{E}}, \mu_{\mathrm{E}}\right)$ be a probability space associated with the Euclidean quantum field (for details see [LHB11, Section 5]). The Hilbert spaces $L^{2}\left(Q_{\mathrm{E}}\right)$ and $L^{2}(Q)$ are related through the family of isometries $\left\{j_{s}\right\}_{s \in \mathbb{R}}$ from $L^{2}\left(\mathbb{R}^{d}\right)$ to $L^{2}\left(\mathbb{R}^{d+1}\right)$ defined by

$$
\widehat{j_{s} f}\left(k, k_{0}\right)=\frac{e^{-i t k_{0}}}{\sqrt{\pi}} \sqrt{\frac{\omega(k)}{\left|k_{0}\right|^{2}+\omega(k)^{2}}} \hat{f}(k)
$$

where $\hat{f}$ denotes the Fourier transform of $f$. Let $\Phi_{\mathrm{E}}\left(j_{s} f\right)$ be a Gaussian random variable on $\left(Q_{\mathrm{E}}, \mu_{\mathrm{E}}\right)$ indexed by $j_{s} f \in L^{2}\left(\mathbb{R}^{d+1}\right)$ with mean zero and covariance

$$
\mathbb{E}_{\mu_{\mathrm{E}}}\left[\Phi_{\mathrm{E}}\left(j_{s} f\right) \Phi_{\mathrm{E}}\left(j_{t} g\right)\right]=\frac{1}{2} \int_{\mathbb{R}^{d}} e^{-|s-t| \omega(k)} \overline{\hat{f}(k)} \hat{g}(k) d k .
$$

Also, let $\left\{J_{s}\right\}_{s \in \mathbb{R}}$ be the family of isometries from $L^{2}(Q)$ to $L^{2}\left(Q_{\mathrm{E}}\right)$ defined by

$$
J_{s}: \phi\left(f_{1}\right) \cdots \phi\left(f_{n}\right):=: \Phi_{\mathrm{E}}\left(j_{s} f_{1}\right) \cdots \Phi_{\mathrm{E}}\left(j_{s} f_{n}\right):
$$

where $: X$ : denotes Wick product of $X$. Then we derive that

$$
\left(J_{s} \Phi, J_{t} \Psi\right)_{L^{2}\left(Q_{\mathrm{E}}\right)}=\left(\Phi, e^{-|t-s| H_{\mathrm{f}}} \Psi\right)_{L^{2}(Q)}
$$

In [HL08] by making use of the process $\left(\sigma_{t}\right)_{t \in \mathbb{R}}$ a functional integral representation of the Pauli-Fierz model with spin $1 / 2$ in non-relativistic quantum electrodynamics was obtained. By a suitable modification we can also construct the functional integral representation of $e^{-t H}$. In fact, the construction in the spin-boson case becomes simpler than in the case of the Pauli-Fierz model, see also [HL08, Remark 6.3] and [LHB11, Section 7.9]. We have the following Feynman-Kac-type formula for the spin-boson Hamiltonian. 
We identify $\mathscr{H}$ as

$$
\mathscr{H} \cong L^{2}\left(\mathbb{Z}_{2} ; L^{2}(Q)\right) \cong L^{2}\left(\mathbb{Z}_{2} \times Q\right)
$$

Proposition 1.2 Let $\Phi, \Psi \in \mathscr{H}$ and $h \in L^{2}\left(\mathbb{R}^{d}\right)$ be real-valued. Then

$$
\begin{aligned}
& (\varepsilon \neq 0) \quad\left(\Phi, e^{-t H} \Psi\right)_{\mathscr{H}}=e^{t} \sum_{\sigma \in \mathbb{Z}_{2}} \mathbb{E}_{P} \mathbb{E}_{\mu_{\mathrm{E}}}\left[\overline{J_{0} \Phi\left(\sigma_{0}\right)} e^{-\alpha \Phi_{\mathrm{E}}\left(\int_{0}^{t} \sigma_{s} j_{s} h d s\right)} \varepsilon^{N_{t}} J_{t} \Psi\left(\sigma_{t}\right)\right] \\
& (\varepsilon=0) \quad\left(\Phi, e^{-t H} \Psi\right)_{\mathscr{H}}=e^{t} \sum_{\sigma \in \mathbb{Z}_{2}} \mathbb{E}_{\mu_{\mathrm{E}}}\left[\overline{J_{0} \Phi(\sigma)} e^{-\alpha \Phi_{\mathrm{E}}\left(\sigma \int_{0}^{t} j_{s} h d s\right)} J_{t} \Psi(\sigma)\right] .
\end{aligned}
$$

Proof: Let $\varepsilon \neq 0$. We see by (1.16) that

$$
H \Psi(\sigma)=\left(H_{\mathrm{f}}+\alpha \phi(h)\right) \Psi(\sigma)-e^{\log \varepsilon} \Psi(-\sigma), \quad \sigma \in \mathbb{Z}_{2} .
$$

Then by [HL08, Theorem 4.11] we have

$$
\left(\Phi, e^{-t H} \Psi\right)_{\mathscr{H}}=e^{t} \sum_{\sigma \in \mathbb{Z}_{2}} \mathbb{E}_{P} \mathbb{E}_{\mu_{\mathrm{E}}}\left[\overline{J_{0} \Phi\left(\sigma_{0}\right)} e^{-\alpha \int_{0}^{t} \sigma_{s} \Phi_{\mathrm{E}}\left(j_{s} f\right) d s+\int_{0}^{t+} \log \varepsilon d N_{s}} J_{t} \Psi\left(\sigma_{t}\right)\right]
$$

Since $\int_{0}^{t+} \log \varepsilon d N_{s}=N_{t} \log \varepsilon,(1.21)$ follows. Next consider the case $\varepsilon=0$. As $\varepsilon \rightarrow 0$ on both sides of (1.21) the integrands over $\left\{N_{t} \geq 1\right\}$ vanish while those on $\left\{N_{t}=0\right\}$ are non-vanishing. Moreover, note that $N_{s}=0, s \leq t$, on $\left\{N_{t}=0\right\}$. Hence (1.22) is obtained by taking the limit

$$
\begin{aligned}
\lim _{\varepsilon \rightarrow 0}\left(\Phi, e^{-t H} \Psi\right)_{\mathscr{H}} & =\lim _{\varepsilon \rightarrow 0} e^{t} \sum_{\sigma \in \mathbb{Z}_{2}} \mathbb{E}_{P} \mathbb{E}_{\mu_{\mathrm{E}}}\left[\overline{J_{0} \Phi\left(\sigma_{0}\right)} e^{-\alpha \Phi_{\mathrm{E}}\left(\int_{0}^{t} \sigma_{s} j_{s} h d s\right)} \varepsilon^{N_{t}} J_{t} \Psi\left(\sigma_{t}\right)\right] \\
& =e^{t} \sum_{\sigma \in \mathbb{Z}_{2}} \mathbb{E}_{\mu_{\mathrm{E}}}\left[\overline{J_{0} \Phi(\sigma)} e^{-\alpha \Phi_{\mathrm{E}}\left(\sigma \int_{0}^{t} j_{s} h d s\right)} J_{t} \Psi(\sigma)\right] .
\end{aligned}
$$

Remark 1.3 By the Feynman-Kac formula (Proposition 1.2) we see that

$$
e^{-t H} \Phi(\sigma)=e^{t} \mathbb{E}_{P}\left[J_{0}^{*} e^{\Phi_{\mathrm{E}}\left(-\alpha \int_{0}^{t} \sigma_{r} j_{r} h d r\right)} J_{t} \Phi\left(\sigma_{t}\right)\right]
$$

for every $\sigma \in \mathbb{Z}_{2}$.

Denote $\mathbb{1}_{\mathscr{H}}=\mathbb{1}_{L^{2}\left(\mathbb{Z}_{2}\right)} \otimes \mathbb{1}_{L^{2}(Q)}$. Using the above proposition we can compute the vacuum expectation of the semigroup $e^{-t H}$, which plays an important role in this paper. 
Corollary 1.4 Let $h \in L^{2}\left(\mathbb{R}^{d}\right)$ be a real-valued function. Then for every $t>0$ it follows that

$$
\left(\mathbb{1}_{\mathscr{H}}, e^{-t H} \mathbb{1}_{\mathscr{H}}\right)=e^{t} \sum_{\sigma \in \mathbb{Z}_{2}} \mathbb{E}_{P}\left[\varepsilon^{N_{t}} e^{\frac{\alpha^{2}}{2} \int_{0}^{t} d r \int_{0}^{t} W\left(N_{r}-N_{s}, r-s\right) d s}\right]
$$

where the pair interaction potential $W$ is given by

$$
W(x, s)=\frac{(-1)^{x}}{2} \int_{\mathbb{R}^{d}} e^{-|s| \omega(k)}|\hat{h}(k)|^{2} d k .
$$

Proof: By Proposition 1.2 we have

$$
\left(\mathbb{1}_{\mathscr{H}}, e^{-t H} \mathbb{1}_{\mathscr{H}}\right)=e^{t} \sum_{\sigma \in \mathbb{Z}_{2}} \mathbb{E}_{P}\left[\varepsilon^{N_{t}} e^{\frac{\alpha^{2}}{2} \int_{0}^{t} d r \int_{0}^{t} W\left(N_{r}+N_{s}, r-s\right) d s}\right]
$$

Since $W\left(N_{r}+N_{s}, r-s\right)=W\left(N_{r}-N_{s}, r-s\right)$, the corollary follows.

Note that equality (1.25) gives the interaction potential

$$
W\left(N_{r}-N_{s}, r-s\right)=\frac{1}{2} \sigma_{r} \sigma_{s} \int_{\mathbb{R}^{d}} e^{-|r-s| \omega(k)}|\hat{h}(k)|^{2} d k
$$

of an infinite range Ising-model on the real line instead of a lattice.

\subsection{Parity symmetry}

It is a known fact that $H_{\mathrm{SB}}$ has a parity symmetry. Let

$$
P=\sigma_{z} \otimes(-1)^{N},
$$

where $N=d \Gamma(\mathbb{1})$ denotes the number operator in $\mathscr{F}$. From $\operatorname{Spec}\left(\sigma_{z}\right)=\{-1,1\}$ and $\operatorname{Spec}(N)=\{0,1,2, \ldots\}$ it follows that $\operatorname{Spec}(P)=\{-1,1\}$. We identify $\mathscr{H}_{\text {with }} \mathscr{F}_{\uparrow} \oplus \mathscr{F}_{\downarrow}$, where $\mathscr{F}_{\uparrow}$ and $\mathscr{F}_{\downarrow}$ are identical copies of $\mathscr{F}$. Then each Pauli matrix $\sigma_{X}=\left[\begin{array}{ll}a & b \\ c & d\end{array}\right]$ acts as

$$
\sigma_{X}\left[\begin{array}{l}
\Psi(+) \\
\Psi(-)
\end{array}\right]=\left[\begin{array}{l}
a \Psi(+)+b \Psi(-) \\
c \Psi(+)+d \Psi(-)
\end{array}\right]
$$

for $\left[\begin{array}{l}\Psi(+) \\ \Psi(-)\end{array}\right] \in \mathscr{F}_{\uparrow} \oplus \mathscr{F}_{\downarrow}$. Furthermore, $\mathscr{F}=\oplus_{n}^{\infty} \mathscr{F}_{n}$ can be decomposed as $\mathscr{F}_{\mathrm{e}} \oplus \mathscr{F}_{\mathrm{o}}$, where $\mathscr{F}_{\mathrm{e}}$ and $\mathscr{F}_{\mathrm{o}}$ denote respectively the subspaces of $\mathscr{F}$ consisting of even and odd numbers of bosons, i.e., $\mathscr{F}_{\mathrm{e}}=\oplus_{m=0}^{\infty} \mathscr{F}_{2 m}$ and $\mathscr{F}_{\mathrm{o}}=\oplus_{m=0}^{\infty} \mathscr{F}_{2 m+1}$. The projections from $\mathscr{F}$ to $\mathscr{F}_{\mathrm{e}}$ and $\mathscr{F}_{\mathrm{o}}$ are denoted by $P_{\mathrm{e}}$ and $P_{\mathrm{o}}$, respectively. Let $\mathscr{H}_{+}=P_{\mathrm{e}} \mathscr{F}_{\uparrow} \oplus P_{\mathrm{o}} \mathscr{F}_{\downarrow}$ and $\mathscr{H}_{-}=P_{\mathrm{o}} \mathscr{F}_{\uparrow} \oplus P_{\mathrm{e}} \mathscr{F}_{\downarrow}$ be subspaces of $\mathscr{F}_{\uparrow} \oplus \mathscr{F}_{\downarrow}$. 
Lemma 1.5 The following properties hold:

(1) The Hilbert space $\mathscr{H}$ can be identified with $\mathscr{H}_{+} \oplus \mathscr{H}_{-}$by the correspondence

$$
\mathscr{F}_{\uparrow} \oplus \mathscr{F}_{\downarrow} \ni\left[\begin{array}{l}
\Psi(+) \\
\Psi(-)
\end{array}\right] \mapsto\left[\begin{array}{l}
\Psi_{\mathrm{e}}(+) \\
\Psi_{\mathrm{o}}(-)
\end{array}\right] \oplus\left[\begin{array}{l}
\Psi_{\mathrm{o}}(+) \\
\Psi_{\mathrm{e}}(-)
\end{array}\right] \in \mathscr{H}_{+} \oplus \mathscr{H}_{-}
$$

where $\Psi_{\mathrm{e}}( \pm)=P_{\mathrm{e}} \Psi( \pm)$ and $\Psi_{\mathrm{o}}( \pm)=P_{\mathrm{o}} \Psi( \pm)$.

(2) It follows that $\left[H_{\mathrm{SB}}, P\right]=0$.

(3) $\mathscr{H}_{ \pm}$is the eigenspace associated with eigenvalue \pm 1 of $P$.

(4) $H_{\mathrm{SB}}$ can be decomposed as $H_{\mathrm{SB}}=H_{\mathrm{SB}}\left\lceil\mathscr{H}_{+} \oplus H_{\mathrm{SB}}\left\lceil\mathscr{H}_{-}\right.\right.$.

Proof: (1) and (2) are straightforward. Let $\Psi=\left[\begin{array}{l}\Psi_{\mathrm{e}}(+) \\ \Psi_{\mathrm{o}}(-)\end{array}\right] \in \mathscr{H}_{+}$. Then $P \Psi=\Psi$ follows by a direct calculation. Thus $\Psi$ is eigenvector of $P$ with eigenvalue +1 . Similarly it follows that $\mathscr{H}_{-}$is the eigenspace associated with eigenvalue -1 of $P$, and (3) also follows. (4) is obtained by a combination of (1), (2) and (3).

Finally, for later use we show some related spin-flip properties.

Lemma 1.6 We have the following properties:

(1) $\left(\Psi, \sigma_{x} \Phi\right)=0$ and $\left(U^{*} \Psi, \sigma_{z} U^{*} \Phi\right)=0$ for any $\Psi, \Phi \in \mathscr{H}_{ \pm}$.

(2) $(\Psi, \phi(f) \Phi)=0$ and $\left(U^{*} \Psi, \phi(f) U^{*} \Phi\right)=0$ for any $\Psi, \Phi \in \mathscr{H}_{ \pm}$.

Proof: (1) It is straightforward to show that $\sigma_{x}$ is a spin-flip transform, i.e.,

$$
\sigma_{x} \mathscr{H}_{ \pm} \subset \mathscr{H}_{\mp}
$$

which gives the first statement. The second statement follows by observing that $U \sigma_{z} U^{*}=\sigma_{x}$. To obtain (2) it is again straightforward to show that

$$
\phi(f)\left(\mathscr{H}_{ \pm} \cap D(\phi(f))\right) \subset \mathscr{H}_{\mp}
$$

The second part follows by $U \phi(f) U^{*}=\phi(f)$. 


\section{Ground state of the spin-boson}

\subsection{Positivity improving semigroup}

In the remainder of this paper we assume that $h \in L^{2}\left(\mathbb{R}^{d}\right)$ is real-valued.

Let $E=\inf \operatorname{Spec}(H)$. We estimate the dimension of $\operatorname{Ker}(H-E)$ for $\varepsilon \neq 0$.

Corollary 2.1 Assume that $\varepsilon \neq 0$. Then $e^{-t H}, t>0$, is a positivity improving semigroup on $L^{2}\left(\mathbb{Z}_{2} \times Q\right)$, i.e., $\left(\Psi, e^{-t H} \Phi\right)>0$ for $\Psi, \Phi \geq 0$ such that $\Psi \not \equiv 0 \not \equiv \Phi$.

Proof: The proof simplifies an argument in [Hir00]. It is trivial that $\left(\Psi, e^{-t H} \Phi\right) \geq 0$, thus it suffices showing that $\left(\Psi, e^{-t H} \Phi\right) \neq 0$. Suppose the contrary. Then we have

$$
\sum_{\sigma \in \mathbb{Z}_{2}} \mathbb{E}_{P}\left[\left(J_{0} \Psi\left(\sigma_{0}\right), e^{\Phi_{\mathrm{E}}\left(-\alpha \int_{0}^{t} \sigma_{s} j_{s} h d s\right)} \varepsilon^{N_{t}} J_{t} \Phi\left(\sigma_{t}\right)\right)_{L^{2}\left(Q_{\mathrm{E}}\right)}\right]=0 .
$$

Since $J_{t}$ is positivity preserving and $e^{\Phi_{\mathrm{E}}\left(-\alpha \int_{0}^{t} \sigma_{s} j_{s} h d s\right)}$ is positive, for $\sigma \in \mathbb{Z}_{2}$ we have

$$
\mathbb{E}_{P}\left[\left(J_{0} \Psi\left(\sigma_{0}\right), e^{\Phi_{\mathrm{E}}\left(-\alpha \int_{0}^{t} \sigma_{s} j_{s} h d s\right)} \varepsilon^{N_{t}} J_{t} \Phi\left(\sigma_{t}\right)\right)_{L^{2}\left(Q_{\mathrm{E}}\right)}\right]=0,
$$

which implies that $\operatorname{supp} J_{0} \Psi\left(\sigma_{0}\right) \cap \operatorname{supp} J_{t} \Phi\left(\sigma_{t}\right)=\emptyset$ a.s. Hence $0=\left(J_{0} \Psi\left(\sigma_{0}\right), J_{t} \Phi\left(\sigma_{t}\right)\right)=$ $\left(\Psi\left(\sigma_{0}\right), e^{-t H_{\mathrm{f}}} \Phi\left(\sigma_{t}\right)\right)$. Since $e^{-t H_{\mathrm{f}}}$ is positivity improving, $\Psi\left(\sigma_{0}\right) \equiv 0$ or $\Phi\left(\sigma_{t}\right) \equiv 0$. This contradicts that $\Psi \not \equiv 0$ and $\Phi \not \equiv 0$, and the claim follows.

\subsection{Existence and uniqueness of ground state}

\subsubsection{The case of $\varepsilon=0$}

Whenever $\varepsilon=0$ the Hamiltonian $H$ is diagonal, i.e., we have

$$
H=\left[\begin{array}{cc}
H_{\mathrm{f}}+\alpha \phi(h) & 0 \\
0 & H_{\mathrm{f}}-\alpha \phi(h)
\end{array}\right]
$$

It is known that $H_{\mathrm{f}}+\alpha \phi(h)$, the Hamiltonian of the van Hove model, has a unique ground state if and only if $\hat{h} / \omega \in L^{2}\left(\mathbb{R}^{d}\right)$, see e.g. [Hir06], which implies that $H$ has a two-fold degenerate ground state if and only if $\hat{h} / \omega \in L^{2}\left(\mathbb{R}^{d}\right)$.

\subsubsection{The case of $\varepsilon \neq 0$}

Next we consider the case of $\varepsilon \neq 0$. Write

$$
\Phi_{T}=e^{-T(H-E)} \mathbb{1}, \quad T \geq 0,
$$


and

$$
\gamma(T)=\frac{\left(\mathbb{1}_{\mathscr{H}}, \Phi_{T}\right)^{2}}{\left\|\Phi_{T}\right\|^{2}}=\frac{\left(\mathbb{1}_{\mathscr{H}}, e^{T H} \mathbb{1}_{\mathscr{H}}\right)^{2}}{\left(\mathbb{1}_{\mathscr{H}}, e^{-2 T H} \mathbb{1}_{\mathscr{H}}\right)}
$$

A known criterion of existence of a ground state is [LHB11, Proposition 6.8].

Proposition 2.2 A ground state of $H$ exists if and only if $\lim _{T \rightarrow \infty} \gamma(T)>0$.

By Corollary 1.4 we have

$$
\begin{aligned}
\left\|\Phi_{T}\right\|^{2} & =e^{2 T E} \sum_{\sigma \in \mathbb{Z}_{2}} \mathbb{E}_{P}\left[\varepsilon^{N_{T}} e^{\frac{\alpha^{2}}{2} \int_{-T}^{T} d t \int_{-T}^{T} W\left(N_{t}-N_{s}, t-s\right) d s}\right], \\
\left(\mathbb{1}_{\mathscr{H}}, \Phi_{T}\right) & =e^{T E} \sum_{\sigma \in \mathbb{Z}_{2}} \mathbb{E}_{P}\left[\varepsilon^{N_{T}} e^{\frac{\alpha^{2}}{2} \int_{0}^{T} d t \int_{0}^{T} W\left(N_{t}-N_{s}, t-s\right) d s}\right] \\
& =e^{T E} \sum_{\sigma \in \mathbb{Z}_{2}} \mathbb{E}_{P}\left[\varepsilon^{N_{T}} e^{\frac{\alpha^{2}}{2} \int_{-T}^{0} d t \int_{-T}^{0} W\left(N_{t}-N_{s}, t-s\right) d s}\right] .
\end{aligned}
$$

The second identity on (2.4) is derived from the reflection symmetry in Proposition 1.1. Note that

$$
\left|\int_{-T}^{0} d t \int_{0}^{T} W\left(N_{t}-N_{s}, t-s\right) d s\right| \leq \frac{1}{2}\left\|\frac{\hat{h}}{\omega}\right\|^{2}
$$

uniformly in $T$ and in the paths.

Theorem 2.3 If $\hat{h} / \omega \in L^{2}\left(\mathbb{R}^{d}\right)$, then $H$ has a ground state and it is unique.

Proof: We write $\int_{-T}^{T} d t \int_{-T}^{T} W d s=\int_{-T}^{0} d t \int_{-T}^{0} W d s+\int_{0}^{T} d t \int_{0}^{T} W d s+2 \int_{-T}^{0} d t \int_{0}^{T} W d s$, and by (2.5) obtain

$$
\left\|\Phi_{T}\right\|^{2} \leq e^{2 T E} \sum_{\sigma \in \mathbb{Z}_{2}} \mathbb{E}_{P}\left[\varepsilon^{N_{T}} e^{\frac{\alpha^{2}}{2}\left(\int_{-T}^{0} d t \int_{-T}^{0} d s W\left(N_{t}-N_{s}, t-s\right)+\int_{0}^{T} d t \int_{0}^{T} d s W\left(N_{t}-N_{s}, t-s\right)+2\|\hat{h} / \omega\|^{2}\right)}\right] .
$$

By the independence of $N_{t}$ and $N_{-s}$, and reflection symmetry of the paths we furthermore obtain that

$$
\begin{aligned}
\left\|\Phi_{T}\right\|^{2} & \leq e^{2 T E} \sum_{\sigma \in \mathbb{Z}_{2}}\left(\mathbb{E}_{P}\left[\varepsilon^{N_{T}} e^{\frac{\alpha^{2}}{2} \int_{0}^{T} d t \int_{0}^{T} d s W\left(N_{t}-N_{s}, t-s\right)}\right]\right)^{2} e^{\frac{\alpha^{2}}{2}\|\hat{h} / \omega\|^{2}} \\
& \leq\left(e^{T E} \sum_{\sigma \in \mathbb{Z}_{2}} \mathbb{E}_{P}\left[\varepsilon^{N_{T}} e^{\frac{\alpha^{2}}{2} \int_{0}^{T} d t \int_{0}^{T} d s W\left(N_{t}-N_{s}, t-s\right)}\right]\right)^{2} e^{\frac{\alpha^{2}}{2}\|\hat{h} / \omega\|^{2}} \\
& =\left(\mathbb{1}_{\mathscr{H}}, \Phi_{T}\right)^{2} e^{\frac{\alpha^{2}}{2}\|\hat{h} / \omega\|^{2}} .
\end{aligned}
$$


Hence $\gamma(T) \geq e^{-\frac{\alpha^{2}}{2}\|\hat{h} / \omega\|^{2}}$ and a ground state $\varphi_{\mathrm{g}}$ of $H$ exists. By Corollary $2.1 \varphi_{\mathrm{g}}$ is strictly positive as a vector in $L^{2}\left(\mathbb{Z}_{2} \times Q\right)$, in particular, it is unique.

We note that the condition $\hat{h} / \omega \in L^{2}\left(\mathbb{R}^{d}\right)$ in Theorem 2.3 is not a mere technicality. This condition will play an essential role throughout below in the definition of a Gibbs measure and the analysis of the ground state properties, see also Section 4.6 below.

By Theorem 2.3 it follows that $H_{\mathrm{SB}}$ also has a unique ground state. As seen above, $\mathscr{H}$ can be decomposed as $\mathscr{H}=\mathscr{H}_{+} \oplus \mathscr{H}_{-}$and $H$ can be reduced by $\mathscr{H}_{ \pm}$.

Corollary 2.4 Let $\varphi_{\mathrm{SB}}$ be the ground state of $H_{\mathrm{SB}}$. Then $\varphi_{\mathrm{SB}} \in \mathscr{H}_{-}$.

Proof: Let $U$ be as in (1.12). Notice that $\varphi_{\mathrm{SB}}=U^{*} \varphi_{\mathrm{g}}$, and thus

$$
\varphi_{\mathrm{SB}}=s-\lim _{T \rightarrow \infty} \frac{U^{*} e^{-T H} \mathbb{1}_{\mathscr{H}}}{\left\|U^{*} e^{-T H} \mathbb{1}_{\mathscr{H}}\right\|}=s-\lim _{T \rightarrow \infty} \frac{e^{-T H_{\mathrm{SB}}} U^{*} \mathbb{1}_{\mathscr{H}}}{\left\|e^{-T H_{\mathrm{SB}}} U^{*} \mathbb{1}_{\mathscr{H}}\right\|} .
$$

The function $\mathbb{1}_{\mathscr{H}} \in L^{2}\left(\mathbb{Z}_{2}\right) \otimes L^{2}(Q)$ corresponds to $\left[\begin{array}{l}\Omega \\ \Omega\end{array}\right] \in \mathscr{F}_{\uparrow} \oplus \mathscr{F}_{\downarrow}$ and

$$
U^{*} \mathbb{1}_{\mathscr{H}}=\frac{1}{2}\left[\begin{array}{cc}
1 & -1 \\
1 & 1
\end{array}\right]\left[\begin{array}{l}
\Omega \\
\Omega
\end{array}\right]=\left[\begin{array}{l}
0 \\
\Omega
\end{array}\right] \in \mathscr{H}_{-} .
$$

Hence by the parity symmetry of $H_{\mathrm{SB}}$ we have

$$
P e^{-T H_{\mathrm{SB}}} U^{*} \mathbb{1}_{\mathscr{H}}=e^{-T H_{\mathrm{SB}}} P U^{*} \mathbb{1}_{\mathscr{H}}=-e^{-T H_{\mathrm{SB}}} U^{*} \mathbb{1}_{\mathscr{H}}
$$

and thus $e^{-T H_{\mathrm{SB}}} U^{*} \mathbb{1}_{\mathscr{H}} \in \mathscr{H}_{-}$. This implies that $\varphi_{\mathrm{SB}} \in \mathscr{H}_{-}$.

Remark 2.5 By Corollary 2.1 the ground state $\varphi_{\mathrm{g}}$ of $H$ overlaps with the non-negative vector $\rho(\sigma, \phi)=\left\{\begin{array}{ll}1, & \sigma=+1 \\ 0, & \sigma=-1\end{array}\right.$ in $L^{2}\left(\mathbb{Z}_{2} \times Q\right)$. Hence $\left(\varphi_{\mathrm{g}}, \rho\right)_{L^{2}\left(\mathbb{Z}_{2} \times Q\right)} \neq 0$ and

$$
\inf \operatorname{Spec}(H)=-\lim _{\beta \rightarrow \infty} \frac{1}{\beta} \log \left(\rho, e^{-\beta H} \rho\right)=-\lim _{\beta \rightarrow \infty} \frac{1}{\beta} \log e^{\beta} \mathbb{E}_{P}\left[\varepsilon^{N_{t}} e^{\frac{\alpha^{2}}{2} \int_{0}^{\beta} d t \int_{0}^{\beta} d s W}\right] .
$$

The expression at the right hand side above was also obtained in [Hir99, Abd12].

\section{Path measure associated with the ground state}

\section{$3.1 \quad \mathbb{Z}_{2}$-valued paths}

In Sections 3.1-3.2 we set $\varepsilon=1$ for simplicity. 
Let $\mathscr{X}=D\left(\mathbb{R} ; \mathbb{Z}_{2}\right)$ be the space of càdlàg paths with values in $\mathbb{Z}_{2}$, and $\mathscr{G}$ the $\sigma$-field generated by cylinder sets. Thus $\sigma:(\Omega, \Sigma, P) \rightarrow(\mathscr{X}, \mathscr{G})$ is an $\mathscr{X}$-valued random variable. We denote its image measure by $\mathcal{W}^{\sigma}$, i.e., $\mathcal{W}^{\sigma}(A)=\sigma_{\text {. }}^{-1}(A)$ for $A \in \mathscr{G}$, and the coordinate process by $\left(X_{t}\right)_{t \in \mathbb{R}}$, i.e., $X_{t}(\omega)=\omega(t)$ for $\omega \in \mathscr{X}$. Hence Proposition 1.2 can be reformulated in terms of $\left(X_{t}\right)_{t \in \mathbb{R}}$ as

$$
\left(\Phi, e^{-t H} \Psi\right)_{\mathscr{H}}=e^{t} \sum_{\sigma \in \mathbb{Z}_{2}} \mathbb{E}_{\mathcal{W}}^{\sigma} \mathbb{E}_{\mu_{\mathrm{E}}}\left[\overline{J_{0} \Phi\left(X_{0}\right)} e^{-\alpha \Phi_{\mathrm{E}}\left(\int_{0}^{t} X_{s} j_{s} h d s\right)} J_{t} \Psi\left(X_{t}\right)\right]
$$

Here $\mathbb{E}_{\mathcal{W}^{\sigma}}=\mathbb{E}_{\mathcal{W}}^{\sigma}$ so that $\mathbb{E}_{\mathcal{W}}^{\sigma}\left[X_{0}=\sigma\right]=1$. Then (1.23) can be converted to the form

$$
e^{-t H} \Phi(\sigma)=e^{t} \mathbb{E}_{\mathcal{W}}^{\sigma}\left[J_{0}^{*} e^{\Phi_{\mathrm{E}}\left(-\alpha \int_{0}^{t} X_{r} j_{r} h d r\right)} J_{t} \Phi\left(\sigma_{t}\right)\right]
$$

for every $\sigma \in \mathbb{Z}_{2}$.

Lemma 3.1 For every $s, t \in \mathbb{R}$ it follows that

$$
\left(\Phi, e^{-t H} \Psi\right)_{\mathscr{H}}=e^{t} \sum_{\sigma \in \mathbb{Z}_{2}} \mathbb{E}_{\mathcal{W}}^{\sigma} \mathbb{E}_{\mu_{\mathrm{E}}}\left[\overline{J_{s} \Phi\left(X_{s}\right)} e^{-\alpha \Phi_{\mathrm{E}}\left(\int_{s}^{s+t} X_{r} j_{r} h d r\right)} J_{s+t} \Psi\left(X_{s+t}\right)\right] .
$$

Proof: By the Trotter product formula $\left(\Phi, e^{-t H} \Psi\right)_{\mathscr{H}}=\lim _{n \rightarrow \infty}\left(\Phi,\left(e^{-\frac{t}{n} H_{0}} e^{-\frac{t}{n} H_{\mathrm{f}}}\right)^{n} \Psi\right)$, and using the fact that $e^{-|t-s| H_{\mathrm{f}}}=J_{t}^{*} J_{s}$ we have

$$
\left(\Phi, e^{-t H} \Psi\right)_{\mathscr{H}}=e^{t} \sum_{\sigma \in \mathbb{Z}_{2}} \mathbb{E}_{P} \mathbb{E}_{\mu_{\mathrm{E}}}\left[\overline{J_{s} \Phi\left(\sigma_{0}\right)} e^{-\alpha \Phi_{\mathrm{E}}\left(\int_{0}^{t} \sigma_{r} j_{s+r} h d r\right)} J_{s+t} \Psi\left(\sigma_{t}\right)\right] .
$$

By the shift invariance of $\left(N_{t}\right)_{t \in \mathbb{R}}$ stated in Proposition 1.1 we have

$$
\left(\Phi, e^{-t H} \Psi\right)_{\mathscr{H}}=e^{t} \sum_{\sigma \in \mathbb{Z}_{2}} \mathbb{E}_{P} \mathbb{E}_{\mu_{\mathrm{E}}}\left[\overline{J_{s} \Phi\left(\sigma_{s}\right)} e^{-\alpha \Phi_{\mathrm{E}}\left(\int_{0}^{t} \sigma_{s+r} j_{s+r} h d r\right)} J_{s+t} \Psi\left(\sigma_{s+t}\right)\right] .
$$

Hence the lemma follows.

Let $\left(\mathbb{Z}_{2}, \mathscr{B}\right)$ be a measurable space with $\sigma$-field $\mathscr{B}=\left\{\emptyset,\{-1\},\{+1\}, \mathbb{Z}_{2}\right\}$. For later use we show a functional integral representation of Euclidean Green functions of the type $\left(\Phi, \mathbb{1}_{A_{0}} e^{-\left(t_{1}-t_{0}\right) H} \mathbb{1}_{A_{1}} e^{-\left(t_{2}-t_{1}\right) H} \cdots e^{-\left(t_{n}-t_{n-1}\right) H} \mathbb{1}_{A_{n}} \Psi\right)$, where $-\infty<t_{0} \leq \ldots \leq t_{n}<$ $\infty$ and $A_{0}, \ldots, A_{n} \in \mathscr{B}$. We see that the operator

$$
Q_{[S, T]}=J_{S}^{*} e^{\Phi_{\mathrm{E}}\left(-\alpha \int_{S}^{T} X_{s} j_{s} h d s\right)} J_{T}: L^{2}(Q) \rightarrow L^{2}(Q)
$$

is bounded. In fact, we have that

$$
\left\|Q_{[S, T]}\right\|_{L^{2}(Q) \rightarrow L^{2}(Q)} \leq\left\|Q_{[S, T]}\right\|_{L^{1}(Q)} \leq e^{\frac{\alpha^{2}}{4}\left\|\int_{S}^{T} X_{s} j_{s} h d s\right\|^{2}}
$$

which was shown in e.g. [HL08, Corollary 4.4]. 
Corollary 3.2 Let $-\infty<t_{0} \leq \ldots \leq t_{n}<\infty$ and $A_{0}, \ldots, A_{n} \in \mathscr{B}$. Then

$$
\begin{aligned}
& (\Phi, \\
& \left.\quad \mathbb{1}_{A_{0}} e^{-\left(t_{1}-t_{0}\right) H} \mathbb{1}_{A_{1}} e^{-\left(t_{2}-t_{1}\right) H} \cdots e^{-\left(t_{n}-t_{n-1}\right) H} \mathbb{1}_{A_{n}} \Psi\right) \\
& \quad=e^{t_{n}-t_{0}} \sum_{\sigma \in \mathbb{Z}_{2}} \mathbb{E}_{\mathcal{W}}^{\sigma} \mathbb{E}_{\mu_{\mathrm{E}}}\left[\left(\prod_{j=0}^{n} \mathbb{1}_{A_{j}}\left(X_{t_{j}}\right)\right) \overline{\Phi\left(X_{t_{0}}\right)} Q_{\left[t_{0}, t_{n}\right]} \Psi\left(X_{t_{n}}\right)\right] .
\end{aligned}
$$

Proof: This is proven by using the Markov properties of both the Poisson process and the Euclidean field, and (1.23). Denote by $\mathscr{N}_{s}=\sigma\left(N_{r}, 0 \leq r \leq s\right)$ the natural filtration of the Poisson process $\left(N_{t}\right)_{t \in \mathbb{R}}$. The Markov property of $\left(N_{t}\right)_{t \in \mathbb{R}}$ and (1.23) yield that

$$
\begin{aligned}
\left(e^{-s H}\right. & \left.\mathbb{1}_{A} e^{-t H} \Phi\right)(\sigma) \\
& =e^{s+t} \mathbb{E}_{P}\left[J_{0}^{*} e^{\Phi_{\mathrm{E}}\left(-\alpha \int_{0}^{s} \sigma_{r} j_{r} h d r\right)} J_{s} \mathbb{1}_{A}\left(\sigma_{s}\right) \mathbb{E}_{P}\left[J_{0}^{*} e^{-\alpha \Phi_{\mathrm{E}}\left(\int_{0}^{t} \sigma_{r+s} j_{r} h d r\right)} J_{t} \Phi\left(\sigma_{t+s}\right) \mid \mathscr{N}_{s}\right]\right] \\
& =e^{s+t} \mathbb{E}_{P}\left[J_{0}^{*} e^{\Phi_{\mathrm{E}}\left(-\alpha \int_{0}^{s} \sigma_{r} j_{r} h d r\right)} J_{s} \mathbb{1}_{A}\left(\sigma_{s}\right) J_{0}^{*} e^{-\alpha \Phi_{\mathrm{E}}\left(\int_{0}^{t} \sigma_{r+s} j_{r} h d r\right)} J_{t} \Phi\left(\sigma_{t+s}\right)\right] \\
& =e^{s+t} \mathbb{E}_{P}\left[J_{0}^{*} e^{-\alpha \Phi_{\mathrm{E}}\left(\int_{0}^{s} \sigma_{r} j_{r} h d r\right)} J_{s} \mathbb{1}_{A}\left(\sigma_{s}\right) J_{0}^{*} e^{-\alpha \Phi_{\mathrm{E}}\left(\int_{0}^{t} \sigma_{r+s} j_{r} h d r\right)} J_{t} \Phi\left(\sigma_{t+s}\right)\right] .
\end{aligned}
$$

Since $J_{0}=U_{-s} J_{s}$, where $U_{s}: L^{2}\left(Q_{\mathrm{E}}\right) \rightarrow L^{2}\left(Q_{\mathrm{E}}\right)$ is the shift operator defined by $U_{s} \Phi_{\mathrm{E}}\left(j_{t_{1}} f_{1}\right) \cdots \Phi_{\mathrm{E}}\left(j_{t_{n}} f_{n}\right)=\Phi_{\mathrm{E}}\left(j_{t_{1}+s} f_{1}\right) \cdots \Phi_{\mathrm{E}}\left(j_{t_{n}+s} f_{n}\right)$, we obtain

$$
\begin{aligned}
& \left(e^{-s H} \mathbb{1}_{A} e^{-t H} \Phi\right)(\sigma) \\
& \quad=e^{s+t} \mathbb{E}_{P}\left[J_{0}^{*} e^{-\alpha \Phi_{\mathrm{E}}\left(\int_{0}^{s} j_{r} \sigma_{r} h d r\right)} J_{s} J_{s}^{*} U_{s} \mathbb{1}_{A}\left(\sigma_{s}\right) e^{-\alpha \Phi_{\mathrm{E}}\left(\int_{0}^{t} \sigma_{r+s} j_{r} h d r\right)} J_{t} \Phi\left(\sigma_{t+s}\right)\right] \\
& \quad=e^{s+t} \mathbb{E}_{P}\left[J_{0}^{*} e^{-\alpha \Phi_{\mathrm{E}}\left(\int_{0}^{s} j_{r} \sigma_{r} h d r\right)} J_{s} J_{s}^{*} \mathbb{1}_{A}\left(\sigma_{s}\right) e^{-\alpha \Phi_{\mathrm{E}}\left(\int_{0}^{t} \sigma_{r+s} j_{r+s} h d r\right)} J_{t+s} \Phi\left(\sigma_{t+s}\right)\right] .
\end{aligned}
$$

Furthermore, by the Markov property of the Euclidean field we can remove the projection $J_{s} J_{s}^{*}$ in $(3.7)$ and obtain

$$
\left(e^{-s H} \mathbb{1}_{A} e^{-t H} \Phi\right)(\sigma)=e^{s+t} \mathbb{E}_{P}\left[J_{0}^{*} e^{-\alpha \Phi_{\mathrm{E}}\left(\int_{0}^{s+t} \sigma_{r} j_{r} h d r\right)} \mathbb{1}_{A}\left(\sigma_{s}\right) J_{t+s} \Phi\left(\sigma_{t+s}\right)\right] .
$$

Hence in terms of $\left(X_{t}\right)_{t \in \mathbb{R}}$ we have

$$
\begin{aligned}
& \left(\Phi, \mathbb{1}_{A_{0}} e^{-s H} \mathbb{1}_{A_{1}} e^{-t H} \mathbb{1}_{A_{2}} \Psi\right) \\
& =e^{s+t} \sum_{\sigma \in \mathbb{Z}_{2}} \mathbb{E}_{\mathcal{W}}^{\sigma}\left[\mathbb{1}_{A_{0}}\left(X_{0}\right) \mathbb{1}_{A_{1}}\left(X_{s}\right) \mathbb{1}_{A_{2}}\left(X_{s+t}\right)\left(J_{0} \Phi\left(X_{0}\right), e^{-\alpha \Phi_{\mathrm{E}}\left(\int_{0}^{s+t} X_{r} j_{r} h d r\right)} J_{t+s} \Psi\left(X_{t+s}\right)\right)\right] .
\end{aligned}
$$

Repeating this procedure, we have

$$
\begin{aligned}
& \left(\Phi, \mathbb{1}_{A_{0}} e^{-\left(t_{1}-t_{0}\right) H} \mathbb{1}_{A_{1}} e^{-\left(t_{2}-t_{1}\right) H} \cdots e^{-\left(t_{n}-t_{n-1}\right) H} \mathbb{1}_{A_{n}} \Psi\right) \\
& \quad=e^{t_{n}-t_{0}} \sum_{\sigma \in \mathbb{Z}_{2}} \mathbb{E}_{\mathcal{W}}^{\sigma} \mathbb{E}_{\mu_{\mathrm{E}}}\left[\left(\prod_{j=0}^{n} \mathbb{1}_{A_{j}}\left(X_{t_{j}-t_{0}}\right)\right) \overline{\Phi\left(X_{0}\right)} Q_{\left[0, t_{n}-t_{0}\right]} \Psi\left(X_{t_{n}-t_{0}}\right)\right] .
\end{aligned}
$$

By the shift invariance of $\sigma_{t}$ (Proposition 1.1) we complete the proof. 
Corollary 3.3 Let $-\infty<t_{0} \leq t_{1} \leq \ldots \leq t_{n}<\infty$ and $A_{0}, \ldots, A_{n} \in \mathscr{B}$. Then

$$
\begin{aligned}
& \left(\mathbb{1}_{A_{0}}, e^{-\left(t_{1}-t_{0}\right) H} \mathbb{1}_{A_{1}} e^{-\left(t_{2}-t_{1}\right) H} \cdots e^{-\left(t_{n}-t_{n-1}\right) H} \mathbb{1}_{A_{n}}\right) \\
& =e^{t_{n}-t_{0}} \sum_{\sigma \in \mathbb{Z}_{2}} \mathbb{E}_{\mathcal{W}}^{\sigma}\left[e^{\frac{\alpha^{2}}{2} \int_{t_{0}}^{t_{n}} d t \int_{t_{0}}^{t_{n}} d s W\left(X_{s}, X_{t}, t-s\right)} \prod_{j=0}^{n} \mathbb{1}_{A_{j}}\left(X_{t_{j}}\right)\right]
\end{aligned}
$$

where $W(x, y, t)=\frac{x y}{2} \int_{\mathbb{R}^{d}} e^{-|t| \omega(k)} \hat{h}(k)^{2} d k$.

Proof: By Corollary 3.2 we have

$$
\operatorname{LHS}(3.9)=e^{t_{n}-t_{0}} \sum_{\sigma \in \mathbb{Z}_{2}} \mathbb{E}_{\mathcal{W}}^{\sigma}\left[\mathbb{E}_{\mu_{\mathrm{E}}}\left[Q_{\left[t_{0}, t_{n}\right]}\right] \prod_{j=0}^{n} \mathbb{1}_{A_{j}}\left(X_{t_{j}}\right)\right] .
$$

Hence the corollary follows.

\subsection{Local weak convergence}

In this section we make the assumption that $\hat{h} / \omega \in L^{2}\left(\mathbb{R}^{d}\right)$, so that there is a unique ground state $\varphi_{\mathrm{g}} \in \mathscr{H}$. Let $\mathscr{G}_{[-T, T]}=\sigma\left(X_{t}, t \in[-T, T]\right)$ be the family of sub- $\sigma$-fields of $\mathscr{G}$ and

$$
\mathcal{G}=\bigcup_{T \geq 0} \mathscr{G}_{[-T, T]}
$$

Let $\overline{\mathcal{G}}=\sigma(\mathcal{G})$. Define the probability measure $\mu_{T}$ on $(\mathscr{X}, \overline{\mathcal{G}})$ by

$$
\mu_{T}(A)=\frac{e^{2 T}}{Z_{T}} \sum_{\sigma \in \mathbb{Z}_{2}} \mathbb{E}_{\mathcal{W}}^{\sigma}\left[\mathbb{1}_{A} e^{\frac{\alpha^{2}}{2} \int_{-T}^{T} d t \int_{-T}^{T} d s W\left(X_{t}, X_{s}, t-s\right)}\right], \quad A \in \overline{\mathcal{G}},
$$

where $Z_{T}$ is the normalizing constant such that $\mu_{T}(\mathscr{X})=1$. This probability measure is a Gibbs measure for the pair interaction potential $W$, indexed by the bounded intervals $[-T, T]$ (see the next section for further details). In this section we show convergence of $\mu_{T}$ to a probability measure $\mu_{\infty}$ in a specific sense when $T \rightarrow \infty$.

Definition 3.4 Let $\mu_{\infty}$ be a probability measure on $(\mathscr{X}, \overline{\mathcal{G}})$, and $\left(T_{n}\right)_{n \in \mathbb{N}} \subset \mathbb{R}$ be any unbounded increasing sequence of positive numbers. The sequence of probability measures $\left(\mu_{T_{n}}\right)_{n \in \mathbb{N}}$ is said to converge to the probability measure $\mu_{\infty}$ in local weak topology whenever $\lim _{n \rightarrow \infty}\left|\mu_{T_{n}}(A)-\mu_{\infty}(A)\right|=0$ for all $A \in \mathscr{G}_{[-t, t]}$ and $t \geq 0$.

By the above definition it is seen that whenever $\mu_{T} \rightarrow \mu_{\infty}$ in local weak sense, we have that

$$
\lim _{T \rightarrow \infty} \mathbb{E}_{\mu_{T}}[f]=\mathbb{E}_{\mu_{\infty}}[f]
$$


for any bounded $\mathscr{G}_{[-t, t]}$-measurable function $f$.

Next we define the finite dimensional distributions indexed by $\Lambda=\left\{t_{0}, \ldots, t_{n}\right\} \subset$ $[-T, T]$ with $t_{0} \leq \ldots \leq t_{n}$. Let

$$
\mu_{T}^{\Lambda}\left(A_{0} \times \cdots \times A_{n}\right)=\frac{e^{2 T}}{Z_{T}} \sum_{\sigma \in \mathbb{Z}_{2}} \mathbb{E}_{\mathcal{W}}^{\sigma}\left[\left(\prod_{j=0}^{n} \mathbb{1}_{A_{j}}\left(X_{t_{j}}\right)\right) e^{\frac{\alpha^{2}}{2} \int_{-T}^{T} d t \int_{-T}^{T} d s W\left(X_{t}, X_{s}, t-s\right)}\right]
$$

be a probability measure on $\left(\mathbb{Z}_{2}^{\Lambda}, \mathscr{B}^{\Lambda}\right)$, where $\mathbb{Z}_{2}^{\Lambda}=\times_{j=1}^{n} \mathbb{Z}_{2}^{t_{j}}$ and $\mathscr{B}^{\Lambda}=\times_{j=1}^{n} \mathscr{B}^{t_{j}}$ for $\Lambda=\left\{t_{1}, \ldots, t_{n}\right\}$, and $\mathbb{Z}_{2}^{t_{j}}$ and $\mathscr{B}^{t_{j}}$ are copies of $\mathbb{Z}_{2}$ and $\mathscr{B}$, respectively. Clearly, $\mathcal{G}$ is a finitely additive family of sets. Define an additive set function on $(\mathscr{X}, \mathcal{G})$ by

$$
\mu(A)=e^{2 E t} e^{2 t} \sum_{\sigma \in \mathbb{Z}_{2}} \mathbb{E}_{\mathcal{W}}^{\sigma}\left[\mathbb{1}_{A}\left(\varphi_{\mathrm{g}}\left(X_{-t}\right), Q_{[-t, t]} \varphi_{\mathrm{g}}\left(X_{t}\right)\right)_{\mathscr{H}}\right], \quad A \in \mathscr{G}_{[-t, t]} .
$$

Note that $\mu(\mathscr{X})=\left(\varphi_{\mathrm{g}}, e^{-2 t(H-E)} \varphi_{\mathrm{g}}\right)=1$.

Lemma 3.5 There exists a unique probability measure $\mu_{\infty}$ on $(\mathscr{X}, \overline{\mathcal{G}})$ such that $\mu_{\infty} \Gamma_{\mathcal{G}}=$ $\mu$. In particular, $\mu_{\infty}(A)=\mu(A)$, for every $A \in \mathscr{G}_{[-t, t]}$ and $t \in \mathbb{R}$.

Proof: Let $\cup_{j=1}^{\infty} A_{j} \in \mathcal{G}$ and $A_{i} \cap A_{j}=\emptyset$ for $i \neq j$. Then there exists $t>0$ such that $\cup_{j=1}^{\infty} A_{j} \in \mathscr{G}_{[-t, t]}$ by the definition of $\mathcal{G}$. Thus by the definition of $\mu$ we have

$$
\mu\left(\cup_{j=1}^{\infty} A_{j}\right)=e^{2 E t} e^{2 t} \sum_{\sigma \in \mathbb{Z}_{2}} \mathbb{E}_{\mathcal{W}}^{\sigma}\left[\mathbb{1}_{\cup_{j=1}^{\infty} A_{j}}\left(\varphi_{\mathrm{g}}\left(X_{-t}\right), Q_{[-t, t]} \varphi_{\mathrm{g}}\left(X_{t}\right)\right)\right]=\sum_{j=1}^{\infty} \mu\left(A_{j}\right)
$$

by the Lebesgue dominated convergence theorem. Hence the set function $\mu$ on $(\mathscr{X}, \mathcal{G})$ is a completely additive measure. Then the Hopf extension theorem implies that there exists a unique probability measure $\mu_{\infty}$ on $(\mathscr{X}, \overline{\mathcal{G}})$ such that $\mu_{\infty}\left\lceil_{\mathcal{G}}=\mu\right.$.

In order to show that $\mu_{T}(A) \rightarrow \mu_{\infty}(A)$ for every $A \in \mathscr{G}_{[-t, t]}$, we define the probability measure $\rho_{T}$ on $\left(\mathscr{X}, \mathscr{G}_{[-T, T]}\right)$ for $A \in \mathscr{G}_{[-t, t]}$ with $t \leq T$ by

$$
\rho_{T}(A)=e^{2 E t} e^{2 t} \sum_{\sigma \in \mathbb{Z}_{2}} \mathbb{E}_{\mathcal{W}}^{\sigma}\left[\mathbb{1}_{A}\left(\frac{\Phi_{T-t}\left(X_{-t}\right)}{\left\|\Phi_{T}\right\|}, Q_{[-t, t]} \frac{\Phi_{T-t}\left(X_{t}\right)}{\left\|\Phi_{T}\right\|}\right)\right] .
$$

The family of probability measures $\rho_{T}^{\Lambda}$ on $\left(\mathbb{Z}_{2}^{\Lambda}, \mathscr{B}^{\Lambda}\right)$ indexed by $\Lambda=\left\{t_{0}, \ldots, t_{n}\right\} \subset$ $[-T, T]$ is defined by

$\rho_{T}^{\Lambda}\left(A_{0} \times \cdots \times A_{n}\right)=e^{2 E t} e^{2 t} \sum_{\sigma \in \mathbb{Z}_{2}} \mathbb{E}_{\mathcal{W}}^{\sigma}\left[\left(\prod_{j=0}^{n} \mathbb{1}_{A_{j}}\left(X_{t_{j}}\right)\right)\left(\frac{\Phi_{T-t}\left(X_{-t}\right)}{\left\|\Phi_{T}\right\|}, Q_{[-t, t]} \frac{\Phi_{T-t}\left(X_{t}\right)}{\left\|\Phi_{T}\right\|}\right)\right]$

for arbitrary $t$ such that $-T \leq-t \leq \ldots \leq t_{0} \leq \ldots \leq t_{n} \leq t \leq T$. To show that $\mu_{T}=\rho_{T}$, we prove that their finite dimensional distributions coincide. 
Lemma 3.6 Let $\Lambda=\left\{t_{0}, t_{1}, \ldots, t_{n}\right\}$ and $A_{0} \times \cdots \times A_{n} \in \mathscr{B}^{\Lambda}$. Then it follows that

$$
\mu_{T}^{\Lambda}\left(A_{0} \times \cdots \times A_{n}\right)=\rho_{T}^{\Lambda}\left(A_{0} \times \cdots \times A_{n}\right) .
$$

In particular, $\rho_{T}^{\Lambda}$ is independent of the choice of $t$.

Proof: By Corollary 3.2 we see that

$$
\mu_{T}^{\Lambda}\left(A_{0} \times \cdots \times A_{n}\right)=\frac{1}{\left\|\Phi_{T}\right\|^{2}}\left(\mathbb{1}_{\mathscr{H}}, e^{-\left(t_{0}+T\right) H} \mathbb{1}_{A_{0}} e^{-\left(t_{1}-t_{0}\right) H} \mathbb{1}_{A_{1}} \cdots \mathbb{1}_{A_{n}} e^{-\left(T-t_{n}\right) H} \mathbb{1}_{\mathscr{H}}\right) .
$$

Hence we have by the definition of $\Phi_{T-t}$ that

$$
\mu_{T}^{\Lambda}\left(A_{0} \times \cdots \times A_{n}\right)=\frac{e^{2 E t}}{\left\|\Phi_{T}\right\|^{2}}\left(\Phi_{T-t}, e^{-\left(t_{0}+t\right) H} \mathbb{1}_{A_{0}} e^{-\left(t_{1}-t_{0}\right) H} \mathbb{1}_{A_{1}} \cdots \mathbb{1}_{A_{n}} e^{-\left(t-t_{n}\right) H} \Phi_{T-t}\right) .
$$

By Corollary 3.2 we have furthermore

$$
\begin{aligned}
\mu_{T}^{\Lambda}\left(A_{0} \times \cdots \times A_{n}\right) & =e^{2 E t} e^{2 t} \sum_{\sigma \in \mathbb{Z}_{2}} \mathbb{E}_{\mathcal{W}}^{\sigma}\left[\prod_{j=0}^{n} \mathbb{1}_{A_{j}}\left(X_{t_{j}}\right)\left(\frac{\Phi_{T-t}}{\left\|\Phi_{T}\right\|}\left(X_{-t}\right), Q_{[-t, t]} \frac{\Phi_{T-t}\left(X_{t}\right)}{\left\|\Phi_{T}\right\|}\right)\right] \\
& =\rho_{T}^{\Lambda}\left(A_{0} \times \cdots \times A_{n}\right) .
\end{aligned}
$$

Thus the lemma follows.

Denote $\mathbb{Z}_{2}^{(-\infty, \infty)}=\left\{\omega:(-\infty, \infty) \rightarrow \mathbb{Z}_{2}\right\}$.

Lemma 3.7 Let $t \leq T$ and $A \in \mathscr{G}_{[-t, t]}$. Then $\mu_{T}(A)=\rho_{T}(A)$.

Proof: It is straightforward to see that the family of probability measures $\mu_{T}^{\Lambda}, \Lambda \subset \mathbb{R}$, on $\left(\mathbb{Z}_{2}^{\Lambda}, \mathscr{B}^{\Lambda}\right)$ with $\# \Lambda<\infty$ satisfies the Kolmogorov consistency condition:

$$
\mu_{T}^{\left\{t_{0}, \ldots, t_{n}, s_{1}, \ldots, s_{m}\right\}}\left(A_{0} \times \cdots \times A_{n} \times \prod^{m} \mathbb{Z}_{2}\right)=\mu_{T}^{\left\{t_{0}, \ldots, t_{n}\right\}}\left(A_{0} \times \cdots \times A_{n}\right) .
$$

Let $\pi_{\Lambda}: \mathbb{Z}_{2}^{(-\infty, \infty)} \rightarrow \mathbb{Z}_{2}^{\Lambda}$ be the projection defined by $\pi_{\Lambda}(\omega)=\left(\omega\left(t_{0}\right), \ldots, \omega\left(t_{n}\right)\right)$ for $\omega \in$ $\mathbb{Z}_{2}^{(-\infty, \infty)}$ and $\Lambda=\left\{t_{0}, \ldots, t_{n}\right\}$. Then $\mathscr{A}_{T}=\left\{\pi_{\Lambda}^{-1}(E) \mid \Lambda \subset[-T, T], \# \Lambda<\infty, E \in \mathscr{B}^{\Lambda}\right\}$ is a finitely additive family of sets. Thus by the Kolmogorov extension theorem there exists a unique probability measure $\mu_{T}^{(-\infty, \infty)}$ on $\left(\mathbb{Z}_{2}^{(-\infty, \infty)}, \sigma\left(\mathscr{A}_{T}\right)\right)$ such that

$$
\mu_{T}^{(-\infty, \infty)}\left(\pi_{\Lambda}^{-1}\left(A_{0} \times \cdots \times A_{n}\right)\right)=\mu_{T}^{\Lambda}\left(A_{0} \times \cdots \times A_{n}\right)
$$

for all $\Lambda \subset[-T, T]$ with $\# \Lambda<\infty$ and $A_{j} \in \mathscr{B}$. Note that $\mathbb{Z}_{2}^{(-\infty, \infty)}=\mathscr{X}$ and $\sigma\left(\mathscr{A}_{T}\right)=\mathscr{G}_{[-T, T]}$ follow. On the other hand, we have

$$
\mu_{T}\left(\pi_{\Lambda}^{-1}\left(A_{0} \times \cdots \times A_{n}\right)\right)=\mu_{T}^{\Lambda}\left(A_{0} \times \cdots \times A_{n}\right),
$$


and $\mu_{T}\left\lceil\mathscr{G}_{[-T, T]}\right.$ is a probability measure on $\left(\mathscr{X}, \mathscr{G}_{[-T, T]}\right)$. Thus the uniqueness of $\mu_{T}^{(-\infty, \infty)}$ satisfying (3.16) implies that $\mu_{T}^{(-\infty, \infty)}=\mu_{T}\left\lceil_{\mathscr{G}_{[-T, T]}}\right.$. Observing that

$$
\mu_{T}^{\Lambda}\left(A_{0} \times \cdots \times A_{n}\right)=\rho_{T}^{\Lambda}\left(A_{0} \times \cdots \times A_{n}\right)=\rho_{T}\left(\pi_{\Lambda}^{-1}\left(A_{0} \times \cdots \times A_{n}\right)\right)
$$

by Lemma 3.6, we also see that $\rho_{T}(A)=\mu_{T}^{(-\infty, \infty)}(A)$ for $A \in \mathscr{G}_{[-t, t]}$ by the uniqueness of $\mu_{T}^{(-\infty, \infty)}$ satisfying (3.16). Thus together with $\mu_{T}^{(-\infty, \infty)}=\mu_{T}\left\lceil\mathscr{G}_{[-T, T]}\right.$ we conclude that $\mu_{T}(A)=\rho_{T}(A)$ for $A \in \mathscr{G}_{[-t, t]}$ and $t \leq T$.

Theorem 3.8 Suppose $\hat{h} / \omega \in L^{2}\left(\mathbb{R}^{d}\right)$. Then the probability measure $\mu_{T}$ on $(\mathscr{X}, \overline{\mathcal{G}})$ converges in local weak sense to $\mu_{\infty}$ as $T \rightarrow \infty$.

Proof: By Lemma 3.7 it suffices to show that $\lim _{T \rightarrow \infty} \rho_{T}(A)=\mu_{\infty}(A)$ for every $A \in$ $\mathscr{G}_{[-T, T]}$. Since $\Phi_{T-t} /\left\|\Phi_{T}\right\| \rightarrow \varphi_{\mathrm{g}}$ strongly in $L^{2}\left(\mathbb{Z}_{2}\right) \otimes L^{2}(Q)$ as $T \rightarrow \infty$, we have $\Phi_{T-t}(\sigma) /\left\|\Phi_{T}\right\| \rightarrow \varphi_{\mathrm{g}}(\sigma)$ for every $\sigma \in \mathbb{Z}_{2}$, strongly in $L^{2}(Q)$. Since $Q_{[-t, t]}$ is a bounded operator, it is seen that

$$
\begin{aligned}
\lim _{T \rightarrow \infty} \rho_{T}(A) & =\lim _{T \rightarrow \infty} e^{2 t} e^{2 E t} \sum_{\sigma \in \mathbb{Z}_{2}} \mathbb{E}_{\mathcal{W}}^{\sigma}\left[\left(\frac{\Phi_{T-t}\left(X_{-t}\right)}{\left\|\Phi_{T}\right\|}, Q_{[-t, t]} \frac{\Phi_{T-t}\left(X_{t}\right)}{\left\|\Phi_{T}\right\|}\right) \mathbb{1}_{A}\right] \\
& =e^{2 t} e^{2 E t} \sum_{\sigma \in \mathbb{Z}_{2}} \mathbb{E}_{\mathcal{W}}^{\sigma}\left[\left(\varphi_{\mathrm{g}}\left(X_{-t}\right), Q_{[-t, t]} \varphi_{\mathrm{g}}\left(X_{t}\right)\right) \mathbb{1}_{A}\right]=\mu_{\infty}(A) .
\end{aligned}
$$

Thus the theorem follows.

\subsection{The case of arbitrary $\varepsilon>0$}

In the case when $\varepsilon \neq 1$ a parallel discussion to the previous section can be made. Since

$$
t H=\varepsilon t\left(-\sigma_{x} \otimes \mathbb{1}+\mathbb{1} \otimes \frac{1}{\varepsilon} H_{\mathrm{f}}+\frac{\alpha}{\varepsilon} \sigma_{z} \otimes \phi(\hat{h})\right),
$$

by replacing $t, h$ and $\omega$ with $\varepsilon t, h / \varepsilon$ and $\omega / \varepsilon$, respectively we have

$$
\left(\Phi, e^{-t H} \Psi\right)_{\mathscr{H}}=e^{\varepsilon t} \sum_{\sigma \in \mathbb{Z}_{2}} \mathbb{E}_{\mathcal{W}}^{\sigma} \mathbb{E}_{\mu_{\mathrm{E}}}\left[\overline{J_{0}^{\varepsilon} \Phi\left(X_{0}\right)} e^{-(\alpha / \varepsilon) \Phi_{\mathrm{E}}\left(\int_{0}^{\varepsilon t} X_{s} j_{s}^{\varepsilon} h d s\right)} J_{t}^{\varepsilon} \Psi\left(X_{\varepsilon t}\right)\right] .
$$

Here $J_{t}^{\varepsilon}$ and $j_{t}^{\varepsilon}$ are defined by $\omega$ replaced by $\omega / \varepsilon$. Thus $j_{s}^{\varepsilon^{*}} j_{t}^{\varepsilon}=e^{-|t-s| \omega / \varepsilon}$ and $J_{s}^{\varepsilon *} J_{t}^{\varepsilon}=$ $e^{-|t-s| H_{\mathrm{f}} / \varepsilon}$. Define the probability measure $\mu_{T}^{\varepsilon}$ on $(\mathscr{X}, \overline{\mathcal{G}})$ by

$$
\mu_{T}^{\varepsilon}(A)=\frac{e^{2 \varepsilon T}}{Z_{\varepsilon T}} \sum_{\sigma \in \mathbb{Z}_{2}} \mathbb{E}_{\mathcal{W}}^{\sigma}\left[\mathbb{1}_{A} e^{\frac{\alpha^{2}}{2} \int_{-T}^{T} d t \int_{-T}^{T} d s W\left(X_{\varepsilon t}, X_{\varepsilon s}, t-s\right)}\right], \quad A \in \overline{\mathcal{G}}
$$


Define also an additive set function on $(\mathscr{X}, \mathcal{G})$ by

$$
\mu^{\varepsilon}(A)=e^{2 E \varepsilon t} e^{2 \varepsilon t} \sum_{\sigma \in \mathbb{Z}_{2}} \mathbb{E}_{\mathcal{W}}^{\sigma}\left[\mathbb{1}_{A}\left(\varphi_{\mathrm{g}}\left(X_{-\varepsilon t}\right), Q_{[-\varepsilon t, \varepsilon t]}^{(\varepsilon)} \varphi_{\mathrm{g}}\left(X_{\varepsilon t}\right)_{\mathscr{H}}\right], \quad A \in \mathscr{G}_{[-\varepsilon t, \varepsilon t]},\right.
$$

where $Q_{[-\varepsilon t, \varepsilon t]}^{(\varepsilon)}=J_{-\varepsilon t}^{\varepsilon *} e^{\Phi_{\mathrm{E}}\left(-(\alpha / \varepsilon) \int_{-\varepsilon t}^{\varepsilon t} X_{s} j_{s}^{\varepsilon} h d s\right)} J_{\varepsilon t^{\varepsilon}}^{\varepsilon}$. In the same way as Lemma 3.5 we see that there exists a unique probability measure $\mu_{\infty}^{\varepsilon}$ on $(\mathscr{X}, \overline{\mathcal{G}})$ such that $\mu_{\infty}^{\varepsilon}\left\lceil_{\mathcal{G}}=\mu^{\varepsilon}\right.$. Furthermore, it can be derived in a similar manner to Theorem 3.8 that

$$
\lim _{T \rightarrow \infty} \mu_{T}^{\varepsilon}(A)=\mu_{\infty}^{\varepsilon}(A), \quad A \in \mathscr{G}_{[-t, t]} .
$$

We summarize this in the theorem below.

Theorem 3.9 Suppose $\hat{h} / \omega \in L^{2}\left(\mathbb{R}^{d}\right)$. Then the probability measure $\mu_{T}^{\varepsilon}$ on $(\mathscr{X}, \overline{\mathcal{G}})$ converges in local weak sense to $\mu_{\infty}^{\varepsilon}$ as $T \rightarrow \infty$.

We also write $\mu_{\mathrm{g}}$ for $\mu_{\infty}^{\varepsilon}$ for notational convenience.

\subsection{Gibbs measure}

In this subsection we show that $\mu_{\mathrm{g}}$ is a Gibbs measure on $(\mathscr{X}, \overline{\mathcal{G}})$. First we give some definitions and basic facts on Gibbs measures needed for this proof.

Let $(\Omega, \mathscr{Y}, Q)$ be a probability space, and $\left(Y_{t}\right)_{t \in \mathbb{R}}$ be a Markov process with càdlàg paths on it. We write $\mathscr{Y}_{T}=\sigma\left(Y_{r}, r \in[-T, T]\right)$ and $\mathscr{T}_{T}=\sigma\left(Y_{r}, r \in[-T, T]^{c}\right)$. Let $\mathscr{V}: \mathbb{R}^{d} \rightarrow \mathbb{R}$ and $\mathscr{W}: \mathbb{R}^{d} \times \mathbb{R}^{d} \times \mathbb{R} \rightarrow \mathbb{R}$ be Borel measurable functions, called external potential and pair potential, respectively. We call $\mathscr{V}$ an admissible external potential whenever

$$
0<\mathbb{E}_{Q}\left[e^{-\int_{I} \mathscr{V}\left(Y_{s}\right) d s}\right]<\infty
$$

for every bounded interval $I \subset \mathbb{R}$. Furthermore, we say that $\mathscr{W}$ is an admissible pair interaction potential whenever

$$
\int_{\mathbb{R}} \sup _{x, y \in \mathbb{R}^{d}}|\mathscr{W}(x, y, s)| d s<\infty .
$$

For the admissible potentials $\mathscr{V}, \mathscr{W}$ and $0<S \leq T$ define the functionals

$$
\begin{aligned}
\mathscr{E}_{T} & =\int_{-T}^{T} \mathscr{V}\left(Y_{t}\right) d t+\left(\int_{\mathbb{R}} d s \int_{-T}^{T} d t+\int_{-T}^{T} d s \int_{\mathbb{R}} d t\right) \mathscr{W}\left(Y_{t}, Y_{s},|t-s|\right), \\
\mathscr{E}_{S, T} & =\int_{-T}^{T} \mathscr{V}\left(Y_{t}\right) d t+\left(\int_{-S}^{S} d s \int_{-T}^{T} d t+\int_{-T}^{T} d s \int_{-S}^{S} d t\right) \mathscr{W}\left(Y_{t}, Y_{s},|t-s|\right) .
\end{aligned}
$$


Also, define $Q_{T}^{Y}$ on $(\Omega, \mathscr{Y})$ for every $Y \in \Omega$ as the unique probability measure such that $\mathbb{E}_{Q_{T}^{Y}}[f g]=\mathbb{E}_{Q}\left[f \mid \mathscr{T}_{T}\right](Y) g(Y)$, for every bounded $\mathscr{Y}_{T}$-measurable function $f$ and every bounded $\mathscr{T}_{T}$-measurable function $g$, i.e.,

$$
Q_{T}^{Y}[A]=\mathbb{E}_{Q}\left[\mathbb{1}_{A} \mid \mathscr{T}_{T}\right](Y) .
$$

Definition 3.10 Suppose that $\mathscr{V}$ and $\mathscr{W}$ are admissible potentials.

(1) A probability measure $P_{T}$ on $(\Omega, \mathscr{Y})$ is called a finite volume Gibbs measure for the interval $[-T, T]$ with respect to the reference measure $Q$ and the potentials $\mathscr{V}$ and $\mathscr{W}$ whenever for all $0<S<T$

(i) $P_{T}\left\lceil\mathscr{Y}_{T} \ll Q\left\lceil\mathscr{Y}_{T}\right.\right.$

(ii) for every bounded $\mathscr{Y}$-measurable function $f$

$$
\mathbb{E}_{P_{T}}\left[f \mid \mathscr{T}_{S}\right](Y)=\frac{\mathbb{E}_{Q_{S}^{Y}}\left[f e^{-\mathscr{E}_{S, T}}\right]}{\mathbb{E}_{Q_{S}^{Y}}\left[e^{-\mathscr{E}_{S, T}}\right]}, \quad P_{T^{\text {-a.s. }}}
$$

(2) A probability measure $P$ is called a Gibbs measure with respect to the reference measure $Q$ and the potentials $\mathscr{V}$ and $\mathscr{W}$ whenever for all $T>0$

(i) $P\left\lceil\mathscr{Y}_{T} \ll Q\left\lceil\mathscr{Y}_{T}\right.\right.$

(ii) for every bounded $\mathscr{Y}$-measurable function $f$

$$
\mathbb{E}_{P}\left[f \mid \mathscr{T}_{T}\right](Y)=\frac{\mathbb{E}_{Q_{T}^{Y}}\left[f e^{-\mathscr{E}_{T}}\right]}{\mathbb{E}_{Q_{t}^{Y}}\left[e^{-\mathscr{E}_{T}}\right]}, \quad P \text {-a.s. }
$$

A sufficient condition for $P_{T}$ to be a finite volume Gibbs measure and $P$ a Gibbs measure is as follows.

Proposition 3.11 Let $\mathscr{V}$ and $\mathscr{W}$ be admissible potentials.

(1) For every $T>0$

$$
d P_{T}=\frac{1}{Z_{T}} e^{-\mathscr{E}_{T, T}} d Q
$$

is a finite volume Gibbs measure for $[-T, T]$, where $Z_{T}$ denotes the normalizing constant.

(2) Suppose that there exists a probability measure $P_{\infty}$ such that $P_{t}(A) \rightarrow P_{\infty}(A)$ as $t \rightarrow \infty$ for all $A \in \mathscr{Y}_{T}$, and $P_{\infty}\left\lceil_{\mathscr{Y}_{T}} \ll Q\left\lceil_{\mathscr{Y}_{T}}\right.\right.$ for every $T$. Then $P_{\infty}$ is a Gibbs measure for the given potentials and reference measure. 
Proof: For (1) see Proposition 4.1, for (2) Proposition 4.2 in [LHB11].

Consider on $\mathbb{Z}_{2}$ the Bernoulli measure

$$
\nu(\sigma)=\frac{1}{2}\left(\delta_{-1}(\sigma)+\delta_{+1}(\sigma)\right), \quad \sigma \in \mathbb{Z}_{2},
$$

and define the probability measure $\mu_{0}$ on $(\mathscr{X}, \overline{\mathcal{G}})$ by

$$
\mu_{0}(A)=\mathbb{E}_{\nu} \mathbb{E}_{\mathcal{W}}^{\sigma}\left[\mathbb{1}_{A}\right], \quad A \in \overline{\mathcal{G}} .
$$

Theorem 3.12 Suppose that $\hat{h} / \omega \in L^{2}\left(\mathbb{R}^{d}\right)$. Then the probability measure $\mu_{\mathrm{g}}$ is a Gibbs measure on $(\mathscr{X}, \overline{\mathcal{G}})$ with respect to reference measure $\mu_{0}$, zero external potential and pair interaction potential

$$
W\left(X_{\varepsilon t}, X_{\varepsilon s},|t-s|\right)=\frac{1}{2} X_{\varepsilon t} X_{\varepsilon s} \int_{\mathbb{R}^{d}} e^{-|t-s| \omega(k)}|\hat{h}(k)|^{2} d k .
$$

Proof: The probability measure $\mu_{T}^{\varepsilon}$ is a finite volume Gibbs measure by part (1) of Proposition 3.11 and (3.18). By Theorem 3.9 we have that $\mu_{T}^{\varepsilon}(A) \rightarrow \mu_{\mathrm{g}}(A)$ as $T \rightarrow \infty$ for every $A \in \mathscr{G}_{[-t, t]}, t \leq T$, and

$$
\begin{aligned}
\mu_{\mathrm{g}}(A) & =e^{2 E \varepsilon t} e^{2 \varepsilon t} \sum_{\sigma \in \mathbb{Z}_{2}} \mathbb{E}_{\mathcal{W}}^{\sigma}\left[\left(\varphi_{\mathrm{g}}\left(X_{-\varepsilon t}\right), Q_{[-\varepsilon t, \varepsilon t]}^{(\varepsilon)} \varphi_{\mathrm{g}}\left(X_{\varepsilon t}\right)\right) \mathbb{1}_{A}\right] \\
& \leq 2 e^{2 E \varepsilon t} e^{2 \varepsilon t}\left\|Q_{[-\varepsilon t, \varepsilon t]}^{(\varepsilon)}\right\|_{L^{1}(Q)} \mu_{0}(A) \leq 2 e^{2 E \varepsilon t} e^{2 \varepsilon t} e^{\alpha^{2} t^{2}\|h\|^{2}} \mu_{0}(A) .
\end{aligned}
$$

This bound is derived from (3.5). Hence $\mu_{\mathrm{g}}\left\lceil\mathscr{Y}_{t} \ll \mu_{0}\left\lceil\mathscr{Y}_{t}\right.\right.$ follows for every $t>0$. Then the theorem follows by part (2) of Proposition 3.11.

\section{Ground state properties}

\subsection{Expectations of functions of the form $\xi(\sigma) F(\phi(f))$}

In this section we use the Gibbs measure obtained above to derive ground state properties of the form (1.2) mentioned in Section 1.1. We start by considering ground state expectations of the form $\left(\varphi_{\mathrm{g}}, \xi(\sigma) F(\phi(f)) \varphi_{\mathrm{g}}\right)$ with suitable functions $F$ and $\xi$ expressed through expectations with respect to the path measure $\mu_{\mathrm{g}}$. By the parity symmetry we know that

$$
\left(\varphi_{\mathrm{g}}, \sigma \varphi_{\mathrm{g}}\right)_{L^{2}\left(\mathbb{Z}_{2} ; L^{2}(Q)\right)}=\left(\varphi_{\mathrm{SB}}, \sigma_{x} \varphi_{\mathrm{SB}}\right)_{\mathbb{C}^{2} \otimes \mathscr{F}}=0
$$




\subsubsection{Expectation of $\xi(\sigma)$}

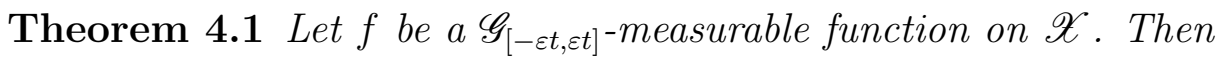

$$
\mathbb{E}_{\mu_{\mathrm{g}}}[f]=e^{2 E \varepsilon t} e^{2 \varepsilon t} \sum_{\sigma \in \mathbb{Z}_{2}} \mathbb{E}_{\mathcal{W}}^{\sigma}\left[\left(\varphi_{\mathrm{g}}\left(X_{-\varepsilon t}\right), Q_{[-\varepsilon t, \varepsilon t]}^{(\varepsilon)} \varphi_{\mathrm{g}}\left(X_{\varepsilon t}\right)\right) f\right] .
$$

Proof: Since for $A \in \mathscr{G}_{[-\varepsilon t, \varepsilon t]}$ we have

$$
\mu_{\mathrm{g}}(A)=e^{2 \varepsilon t} e^{2 E \varepsilon t} \sum_{\sigma \in \mathbb{Z}_{2}} \mathbb{E}_{\mathcal{W}}^{\sigma}\left[\left(\varphi_{\mathrm{g}}\left(X_{-\varepsilon t}\right), Q_{[-\varepsilon t, \varepsilon t]}^{(\varepsilon)} \varphi_{\mathrm{g}}\left(X_{\varepsilon t}\right)\right) \mathbb{1}_{A}\right]
$$

(4.2) follows.

An immediate consequence of Theorem 4.1 is the following.

Corollary 4.2 Let $f_{j}: \mathbb{Z}_{2} \rightarrow \mathbb{C}, j=0, \ldots, n$, be bounded functions. Then

$$
\mathbb{E}_{\mu_{\mathrm{g}}}\left[\prod_{j=0}^{n} f_{j}\left(X_{\varepsilon t_{j}}\right)\right]=\left(\varphi_{\mathrm{g}}, f_{0} e^{-\left(t_{1}-t_{0}\right)(H-E)} f_{1} \cdots e^{-\left(t_{n}-t_{n-1}\right)(H-E)} f_{n} \varphi_{\mathrm{g}}\right) .
$$

In particular, we have for all bounded functions $\xi, f$ and $g$ that

$$
\begin{aligned}
\mathbb{E}_{\mu_{\mathrm{g}}}\left[\xi\left(X_{0}\right)\right] & =\left(\varphi_{\mathrm{g}}, \xi(\sigma) \varphi_{\mathrm{g}}\right), \\
\mathbb{E}_{\mu_{\mathrm{g}}}\left[f\left(X_{t}\right) g\left(X_{s}\right)\right] & =\left(f(\sigma) \varphi_{\mathrm{g}}, e^{-|t-s|(H-E)} g(\sigma) \varphi_{\mathrm{g}}\right) .
\end{aligned}
$$

Proof: For $A_{j} \in \mathscr{B}, j=0,1, \ldots, n$, it follows that

$$
\begin{aligned}
\mathbb{E}_{\mu_{\mathrm{g}}}\left[\prod_{j=0}^{n} \mathbb{1}_{A_{j}}\left(X_{\varepsilon t_{j}}\right)\right] & =e^{2 \varepsilon t} e^{2 E \varepsilon t} \sum_{\sigma \in \mathbb{Z}_{2}} \mathbb{E}_{\mathcal{W}}^{\sigma}\left[\left(\varphi_{\mathrm{g}}\left(X_{-\varepsilon t}\right), Q_{[-\varepsilon t, \varepsilon t]}^{(\varepsilon)} \varphi_{\mathrm{g}}\left(X_{\varepsilon t}\right)\right) \prod_{j=0}^{n} \mathbb{1}_{A_{j}}\left(X_{\varepsilon t_{j}}\right)\right] \\
& =\left(\varphi_{\mathrm{g}}, \mathbb{1}_{A_{0}} e^{-\left(t_{1}-t_{0}\right)(H-E)} \mathbb{1}_{A_{1}} \cdots e^{-\left(t_{n}-t_{n-1}\right)(H-E)} \mathbb{1}_{A_{n}} \varphi_{\mathrm{g}}\right) .
\end{aligned}
$$

Hence (4.3) is obtained.

\subsubsection{Expectation of $\xi(\sigma) F(\phi(f))$}

Lemma 4.3 Let $F$ be a real-valued bounded function on $\mathbb{R}, f \in L^{2}\left(\mathbb{R}^{d}\right)$ and $\xi: \mathbb{Z}_{2} \rightarrow \mathbb{C}$ be a bounded function. Then

$$
\begin{aligned}
& \left(e^{-T H} \mathbb{1}_{\mathscr{H}}, \xi(\sigma) F(\phi(f)) e^{-T H} \mathbb{1}_{\mathscr{H}}\right) \\
& \quad=e^{2 \varepsilon T} \sum_{\sigma \in \mathbb{Z}_{2}} \mathbb{E}_{\mathcal{W}}^{\sigma} \mathbb{E}_{\mu_{\mathrm{E}}}\left[\xi\left(X_{0}\right) e^{-\frac{\alpha}{\varepsilon} \Phi_{\mathrm{E}}\left(\int_{-\varepsilon T}^{\varepsilon T} X_{s} j_{s} h d s\right)} F\left(\Phi_{\mathrm{E}}\left(j_{0} f\right)\right)\right] .
\end{aligned}
$$


Proof: By (3.2) we have

$\left(e^{-T H} \xi(\sigma) F(\phi(f)) e^{-T H} \mathbb{1}_{\mathscr{H}}\right)(\sigma)=e^{2 \varepsilon T} \mathbb{E}_{\mathcal{W}}^{\sigma}\left[Q_{[-\varepsilon T, 0]}^{(\varepsilon)} \xi\left(X_{0}\right) F(\phi(f)) \mathbb{E}_{\mathcal{W}}^{X_{0}}\left[Q_{[0, \varepsilon T]}^{(\varepsilon)} \mathbb{1}_{\mathscr{H}}\left(X_{\varepsilon T}\right)\right]\right]$.

Here $Q_{[S, T]}^{(\varepsilon)}=J_{S}^{\varepsilon *} e^{\Phi_{\mathrm{E}}\left(-\frac{\alpha}{\varepsilon} \int_{S}^{T} X_{s} j_{s}^{\varepsilon} h d s\right)} J_{T}^{\varepsilon}$. Then in a similar manner to the proof of Corollary 3.2 the lemma follows from the Markov property of $\left(N_{t}\right)_{t \in \mathbb{R}}$.

Theorem 4.4 Let $\hat{h} / \omega \in L^{2}\left(\mathbb{R}^{d}\right), f \in L^{2}\left(\mathbb{R}^{d}\right)$ be real-valued, $\xi: \mathbb{Z}_{2} \rightarrow \mathbb{C}$ be a bounded function, and $\beta \in \mathbb{R}$. Then

$$
\left(\varphi_{\mathrm{g}}, \xi(\sigma) e^{i \beta \phi(f)} \varphi_{\mathrm{g}}\right)=e^{-\frac{\beta^{2}}{4}\|f\|^{2}} \mathbb{E}_{\mu_{\mathrm{g}}}\left[\xi\left(X_{0}\right) e^{i \beta K(f)}\right]
$$

where $K(f)$ is a random variable on $(\mathscr{X}, \overline{\mathcal{G}})$ given by

$$
K(f)=\frac{\alpha}{2} \int_{-\infty}^{\infty}\left(e^{-|r| \omega} \hat{h}, \hat{f}\right) X_{\varepsilon r} d r .
$$

Proof: Note that

$$
\left(\varphi_{\mathrm{g}}, \xi(\sigma) e^{i \beta \phi(f)} \varphi_{\mathrm{g}}\right)=\lim _{T \rightarrow \infty}\left(\frac{\Phi_{T}}{\left\|\Phi_{T}\right\|}, \xi(\sigma) e^{i \beta \phi(f)} \frac{\Phi_{T}}{\left\|\Phi_{T}\right\|}\right)
$$

and by Lemma 4.3 we see that

$$
\left(\frac{\Phi_{T}}{\left\|\Phi_{T}\right\|}, \xi(\sigma) e^{i \beta \phi(f)} \frac{\Phi_{T}}{\left\|\Phi_{T}\right\|}\right)=\frac{1}{Z_{\varepsilon T}} e^{2 \varepsilon T} \sum_{\sigma \in \mathbb{Z}_{2}} \mathbb{E}_{\mathcal{W}}^{\sigma} \mathbb{E}_{\mu_{\mathrm{E}}}\left[\xi\left(X_{0}\right) e^{-\frac{\alpha}{\varepsilon} \Phi_{\mathrm{E}}\left(\int_{-\varepsilon T}^{\varepsilon T} X_{s} j_{s} h d s\right)} e^{i \beta \Phi_{\mathrm{E}}\left(j_{0} f\right)}\right]
$$

The expectation with respect to $\mu_{\mathrm{E}}$ can be computed explicitly and thus

$$
\begin{aligned}
& \left(\varphi_{\mathrm{g}}, \xi(\sigma) e^{i \beta \phi(f)} \varphi_{\mathrm{g}}\right) \\
& =\lim _{T \rightarrow \infty} e^{-\frac{\beta^{2}}{4}\|f\|^{2}} \frac{1}{Z_{\varepsilon T}} e^{2 T} \sum_{\sigma \in \mathbb{Z}_{2}} \mathbb{E}_{\mathcal{W}}^{\sigma}\left[\xi\left(X_{0}\right) e^{\frac{\alpha^{2}}{2} \int_{-T}^{T} d t \int_{-T}^{T} W\left(X_{\varepsilon t}, X_{\varepsilon s}, t-s\right) d s} e^{i \beta \int_{-T}^{T}\left(e^{-|s| \omega} \hat{h}, \hat{f}\right) X_{\varepsilon s} d s}\right] \\
& =\lim _{T \rightarrow \infty} e^{-\frac{\beta^{2}}{4}\|f\|^{2}} \mathbb{E}_{\mu_{T}^{\varepsilon}}\left[\xi\left(X_{0}\right) e^{i \beta \int_{-T}^{T}\left(e^{-|s| \omega} \hat{h}, \hat{f}\right) X_{\varepsilon s} d s}\right] .
\end{aligned}
$$

Notice that $\left|\int_{-\infty}^{\infty} X_{\varepsilon s}\left(e^{-|s| \omega} \hat{h}, \hat{f}\right) d s\right| \leq 2\|\hat{h} / \omega\|\|\hat{f}\|<\infty$. By the local weak convergence of $\mu_{T}$ and a similar telescoping as in the proof of Theorem 4.16 below, we obtain the desired result.

By using Theorem 4.4 the functionals $\left(\varphi_{\mathrm{g}}, \xi(\sigma) F(\phi(f)) \varphi_{\mathrm{g}}\right)$ can be represented in terms of averages with respect to the path measure $\mu_{\mathrm{g}}$. Consider the case when $F$ is a polynomial or a Schwartz test function. We will show in Corollary 4.18 below that $\varphi_{\mathrm{g}} \in D\left(e^{+\beta N}\right)$ for all $\beta>0$, thus $\varphi_{\mathrm{g}} \in D\left(\phi(f)^{n}\right)$ for every $n \in \mathbb{N}$. 
Corollary 4.5 Let $\hat{h} / \omega \in L^{2}\left(\mathbb{R}^{d}\right), f \in L^{2}\left(\mathbb{R}^{d}\right)$ be real-valued, and $\xi: \mathbb{Z}_{2} \rightarrow \mathbb{C}$ a bounded function. Also, let $h_{n}(x)=(-1)^{n} e^{x^{2} / 2} \frac{d^{n}}{d x^{n}} e^{-x^{2} / 2}$ be the Hermite polynomial of order $n$. Then

$$
\left(\varphi_{\mathrm{g}}, \xi(\sigma) \phi(f)^{n} \varphi_{\mathrm{g}}\right)=i^{n} \mathbb{E}_{\mu_{\mathrm{g}}}\left[\xi\left(X_{0}\right) h_{n}\left(\frac{-i K(f)}{\|f\| 2^{-1 / 2}}\right)\right]\left(\|f\| 2^{-1 / 2}\right)^{n}, \quad n \in \mathbb{N} .
$$

Proof: We have

$$
e^{-\beta^{2}\|f\|^{2} / 4} e^{i \beta K(f)}=\sum_{n=0}^{\infty} h_{n}\left(\frac{-i K(f)}{\|f\| 2^{-1 / 2}}\right) \frac{\left(-\beta\|f\| 2^{-1 / 2}\right)^{n}}{n !} .
$$

Hence

$$
\frac{1}{i^{n}} \frac{d^{n}}{d \beta^{n}} e^{-\beta^{2}\|f\|^{2} / 4} e^{i \beta K(f)} \Gamma_{\beta=0}=i^{n} h_{n}\left(\frac{-i K(f)}{\|f\| 2^{-1 / 2}}\right)\left(\|f\| 2^{-1 / 2}\right)^{n}
$$

follows. By (4.10) and the computation

$$
\left(\varphi_{\mathrm{g}}, \xi(\sigma) \phi(f)^{n} \varphi_{\mathrm{g}}\right)=\frac{1}{i^{n}} \frac{d^{n}}{d \beta^{n}} e^{-\frac{\beta^{2}}{4}\|f\|^{2}} \mathbb{E}_{\mu_{\mathrm{g}}}\left[\xi\left(X_{0}\right) e^{i \beta K(f)}\right] \Gamma_{\beta=0},
$$

we obtain (4.8).

In the next corollary we give the path integral representation of $\left(\varphi_{\mathrm{g}}, \xi(\sigma) F(\phi(f)) \varphi_{\mathrm{g}}\right)$ for $F \in \mathscr{S}(\mathbb{R})$, where $\mathscr{S}(\mathbb{R})$ denotes the space of rapidly decreasing, infinitely many times differentiable functions on $\mathbb{R}$.

Corollary 4.6 Let $\hat{h} / \omega \in L^{2}\left(\mathbb{R}^{d}\right), f \in L^{2}\left(\mathbb{R}^{d}\right)$ be real-valued, $F \in \mathscr{S}(\mathbb{R})$, and $\xi: \mathbb{Z}_{2} \rightarrow$ $\mathbb{C}$ a bounded function. Then

$$
\left(\varphi_{\mathrm{g}}, \xi(\sigma) F(\phi(f)) \varphi_{\mathrm{g}}\right)=\mathbb{E}_{\mu_{\mathrm{g}}}\left[\xi\left(X_{0}\right) G(K(f))\right]
$$

where $G=\check{F} * \check{g}$ and $g(\beta)=e^{-\beta^{2}\|f\|^{2} / 4}$.

Proof: Since $F(\phi(f))=\frac{1}{\sqrt{2 \pi}} \int_{-\infty}^{\infty} \check{F}(\beta) e^{i \beta \phi(f)} d \beta$, we have

$$
\left(\varphi_{\mathrm{g}}, \xi(\sigma) F(\phi(f)) \varphi_{\mathrm{g}}\right)=\frac{1}{\sqrt{2 \pi}} \int_{-\infty}^{\infty} \check{F}(\beta) e^{-\frac{\beta^{2}}{4}\|f\|^{2}} \mathbb{E}_{\mu_{\mathrm{g}}}\left[\xi\left(X_{0}\right) e^{i \beta K(f)}\right] d \beta .
$$

Thus the corollary follows. 


\subsubsection{Field fluctuations in the ground state}

The field fluctuations in the ground state are defined for every real-valued function $f \in L^{2}\left(\mathbb{R}^{d}\right)$ by

$$
F_{\alpha}(f)=\left(\varphi_{\mathrm{g}}, \phi(f)^{2} \varphi_{\mathrm{g}}\right)-\left(\varphi_{\mathrm{g}}, \phi(f) \varphi_{\mathrm{g}}\right)^{2}
$$

More generally, we also consider fluctuations of the form

$$
G_{\alpha}(f)=\left(\varphi_{\mathrm{g}},(\sigma \phi(f))^{2} \varphi_{\mathrm{g}}\right)-\left(\varphi_{\mathrm{g}}, \sigma \phi(f) \varphi_{\mathrm{g}}\right)^{2}
$$

Corollary 4.7 Let $\hat{h} / \omega \in L^{2}\left(\mathbb{R}^{d}\right)$ and $f \in L^{2}\left(\mathbb{R}^{d}\right)$ be a real-valued function. Then

(1) $\left(\varphi_{\mathrm{g}}, \sigma \phi(f) \varphi_{\mathrm{g}}\right)=\mathbb{E}_{\mu_{\mathrm{g}}}\left[X_{0} K(f)\right]$,

(2) $\left(\varphi_{\mathrm{g}},(\sigma \phi(f))^{2} \varphi_{\mathrm{g}}\right)=\mathbb{E}_{\mu_{\mathrm{g}}}\left[\left(X_{0} K(f)\right)^{2}\right]+\frac{1}{2}\|f\|^{2}$.

In particular,

(3) $G_{\alpha}(f)=\mathbb{E}_{\mu_{\mathrm{g}}}\left[\left(X_{0} K(f)\right)^{2}\right]-\left(\mathbb{E}_{\mu_{\mathrm{g}}}\left[X_{0} K(f)\right]\right)^{2}+\frac{1}{2}\|f\|^{2}$,

(4) $\left(\varphi_{\mathrm{g}}, \phi(f) \varphi_{\mathrm{g}}\right)=0$ and $F_{\alpha}(f)=\left(\varphi_{\mathrm{g}}, \phi(f)^{2} \varphi_{\mathrm{g}}\right)=\mathbb{E}_{\mu_{\mathrm{g}}}\left[K(f)^{2}\right]+\frac{1}{2}\|f\|^{2}$,

(5) whenever $f \not \equiv 0$, we furthermore have that (i) $F_{\alpha}(f)>0$ and $F_{\alpha}(f) \geq F_{0}(f)$, (ii) $G_{\alpha}(f)>0$ and $G_{\alpha}(f) \geq G_{0}(f)$.

Proof: Statements (1)-(3) easily follow from Corollary 4.5, which imply (4) for $\sigma=1$. Using Schwarz inequality, we obtain (5ii), while (5i) is clear by (4).

Note that to prove (1)-(2) of Corollary 4.7 we can proceed, alternatively, to derive first the equality $\left(\varphi_{\mathrm{g}}, \phi(f) \varphi_{\mathrm{g}}\right)=\mathbb{E}_{\mu_{\mathrm{g}}}[K(f)]$ by using Corollary 4.5 , and from $\mathbb{E}_{\mu_{\mathrm{g}}}\left[X_{s}\right]=$ $\left(\varphi_{\mathrm{g}}, \sigma \varphi_{\mathrm{g}}\right)$ to further obtain that

$$
\left(\varphi_{\mathrm{g}}, \phi(f) \varphi_{\mathrm{g}}\right)=-\frac{\alpha}{2}(\hat{h} / \omega, \hat{f})\left(\varphi_{\mathrm{g}}, \sigma \varphi_{\mathrm{g}}\right)=0
$$

Notice that $X_{0}^{2}=1$. Thus in Corollary 4.7 we have equivalently $\mathbb{E}_{\mu_{\mathrm{g}}}\left[\left(X_{0} K(f)\right)^{2}\right]=$ $\mathbb{E}_{\mu_{\mathrm{g}}}\left[K(f)^{2}\right]$. 


\subsection{Gaussian decay and exponential moments of the field op- erator}

\subsubsection{Gaussian decay of the field operator}

In this section we show that $\left(\varphi_{\mathrm{g}}, e^{\beta \phi(f)^{2}} \varphi_{\mathrm{g}}\right)<\infty$ for some $\beta>0$.

Lemma 4.8 Let $\hat{h} / \omega \in L^{2}\left(\mathbb{R}^{d}\right)$ and $f \in L^{2}\left(\mathbb{R}^{d}\right)$ be a real-valued function. Then for $\beta>0$ we have

$$
\left(\varphi_{\mathrm{g}}, e^{-\beta \phi(f)^{2}} \varphi_{\mathrm{g}}\right)=\frac{1}{\sqrt{1+\beta\|f\|^{2}}} \mathbb{E}_{\mu_{\mathrm{g}}}\left[e^{-\frac{\beta K^{2}(f)}{1+\beta\|f\|^{2}}}\right]
$$

Proof: By Theorem 4.4 we see that

$$
\begin{aligned}
\left(\varphi_{\mathrm{g}}, e^{-\left(\beta^{2} / 2\right) \phi(f)^{2}} \varphi_{\mathrm{g}}\right) & =\frac{1}{\sqrt{2 \pi}} \int_{\mathbb{R}} e^{-\beta^{2} / 2}\left(\varphi_{\mathrm{g}}, e^{i \beta \phi(f)} \varphi_{\mathrm{g}}\right) d \beta \\
& =\frac{1}{\sqrt{2 \pi}} \int_{\mathbb{R}} e^{-\beta^{2} / 2} e^{-\beta^{2}\|f\|^{2} / 4} \mathbb{E}_{\mu_{\mathrm{g}}}\left[e^{i \beta K(f)}\right] d \beta \\
& =\frac{1}{\sqrt{1+\beta^{2}\|f\|^{2} / 2}} \mathbb{E}_{\mu_{\mathrm{g}}}\left[e^{-\frac{\beta^{2} K^{2}(f) / 2}{1+\beta^{2}\|f\|^{2} / 2}}\right]
\end{aligned}
$$

Replacing $\beta^{2} / 2$ by $\beta$ completes the proof of the lemma.

Theorem 4.9 Let $\hat{h} / \omega \in L^{2}\left(\mathbb{R}^{d}\right)$ and $f \in L^{2}\left(\mathbb{R}^{d}\right)$ be a real-valued function. If $-\infty<$ $\beta<1 /\|f\|^{2}$, then $\varphi_{\mathrm{g}} \in D\left(e^{(\beta / 2) \phi(f)^{2}}\right)$ and

$$
\left\|e^{(\beta / 2) \phi(f)^{2}} \varphi_{\mathrm{g}}\right\|^{2}=\frac{1}{\sqrt{1-\beta\|f\|^{2}}} \mathbb{E}_{\mu_{\mathrm{g}}}\left[e^{\frac{\beta K^{2}(f)}{1-\beta\|f\|^{2}}}\right] .
$$

Proof: The proof is a modification of [Hir04, Theorem 10.12].

Let $B=\left\{z \in \mathbb{C}|| z \mid<1 /\|f\|^{2}\right\}, \mathbb{C}_{+}=\{z \mid \Re z>0\}$ and $\mathbb{C}_{-}=\{z \mid \Re z<0\}$. Consider

$$
\rho(z)=\frac{1}{\sqrt{1+z\|f\|^{2}}} \mathbb{E}_{\mu_{\mathrm{g}}}\left[e^{-\frac{z K^{2}(f)}{1+z\|f\|^{2}}}\right],
$$

for $z>0$. Then $\rho(z)$ can be analytically continued to $\mathbb{C}_{+} \cup B$, since $|K(f)| \leq$ $|\alpha|\|f\|\|\hat{h} / \omega\|$ uniformly in paths. We denote this extension by $\bar{\rho}(z)$. Let $w \in \mathbb{R} \cap B$ and consider the ball $B_{\delta}(w)=\{z \in \mathbb{C}|| z-w \mid<\delta\}$. Take any $\delta<1 /\|f\|^{2}$ such that for $w$ we have $B_{\delta}(w) \cap \mathbb{C}_{-} \cap B \neq \emptyset$. We expand $\bar{\rho}(z)$ as

$$
\bar{\rho}(z)=\sum_{n=0}^{\infty}(z-w)^{n} b_{n}(w), \quad z \in B_{\delta}(w) \cap B
$$


and get in particular

$$
\bar{\rho}(z)=\sum_{n=0}^{\infty}|z-w|^{n}\left|b_{n}(w)\right|<\infty
$$

for $z \in B_{\delta}(w) \cap B$. On the other hand, $\mathbb{C}_{+} \ni z \mapsto\left(\varphi_{\mathrm{g}}, e^{-z \phi(f)^{2}} \varphi_{\mathrm{g}}\right) \in \mathbb{C}$ is differentiable on $\mathbb{C}_{+}$, since $\varphi_{\mathrm{g}} \in D\left(\phi(f)^{2}\right)$, and is thus analytic on $\mathbb{C}_{+}$. We have

$$
\left(\varphi_{\mathrm{g}}, e^{-z \phi(f)^{2}} \varphi_{\mathrm{g}}\right)=\sum_{n=0}^{\infty}(z-w)^{n} \frac{1}{n !} \int_{0}^{\infty}(-\lambda)^{n} e^{-w \lambda} d E_{\lambda}, \quad z \in \mathbb{C}_{+} .
$$

Here $E_{\lambda}$ denotes the spectral measure of $\frac{1}{2} \phi(f)^{2}$ with respect to $\varphi_{\mathrm{g}}$. Comparing (4.19), (4.21) and $\bar{\rho}(z)=\left(\varphi_{\mathrm{g}}, e^{-z \phi(f)^{2}} \varphi_{\mathrm{g}}\right)$ for $z \in \mathbb{C}_{+}$, we conclude that

$$
b_{n}(w)=\frac{1}{n !} \int_{0}^{\infty}(-\lambda)^{n} e^{-w \lambda} d E_{\lambda} .
$$

Substituting (4.22) into (4.19) we have

$$
\bar{\rho}(z)=\sum_{n=0}^{\infty}(z-w)^{n} \frac{1}{n !} \int_{0}^{\infty}(-\lambda)^{n} e^{-w \lambda} d E_{\lambda}, \quad z \in B_{\delta}(w) \cap B
$$

where the right hand side is absolutely convergent for every $z \in B_{\delta}(w) \cap B$. Thus by (4.20) for $z \in B_{\delta}(w) \cap B \cap \mathbb{R}$ we have

$\int_{0}^{M} e^{-z \lambda} d E_{\lambda} \leq \sum_{n=0}^{\infty} \frac{|z-w|^{n}}{n !}\left|\int_{0}^{M}(-\lambda)^{n} e^{-w \lambda} d E_{\lambda}\right| \leq \sum_{n=0}^{\infty} \frac{|z-w|^{n}}{n !}\left|\int_{0}^{\infty}(-\lambda)^{n} e^{-w \lambda} d E_{\lambda}\right|<\infty$ which implies that $\lim _{M \rightarrow \infty} \int_{0}^{M} e^{-z \lambda} d E_{\lambda}<\infty$ for these $z$. The monotone convergence theorem then gives $\int_{0}^{\infty} e^{-z \lambda} d E_{\lambda}<\infty$, hence $\varphi_{\mathrm{g}} \in D\left(e^{-(z / 2) \phi(f)^{2}}\right)$ and

$$
\left\|e^{-(z / 2) \phi(f)^{2}} \varphi_{\mathrm{g}}\right\|^{2}=\bar{\rho}(z), \quad z \in B_{\delta}(w) \cap B \cap \mathbb{R} .
$$

Since for every $\delta<1 /\|f\|^{2}$ there exists $w \in \mathbb{R} \cap B$ such that $\mathbb{C}_{-} \cap B \cap B_{\delta}(w) \neq \emptyset$, the proof of the theorem is complete.

From Theorem 4.9 it is immediate to get the limit of $\left\|e^{(\beta / 2) \phi(f)^{2}} \varphi_{\mathrm{g}}\right\|$.

Corollary 4.10 Suppose that $\hat{h} / \omega \in L^{2}\left(\mathbb{R}^{d}\right)$ and $f \in L^{2}\left(\mathbb{R}^{d}\right)$ is a real-valued function. Then

$$
\lim _{\beta \uparrow 1 /\|f\|^{2}}\left\|e^{(\beta / 2) \phi(f)^{2}} \varphi_{\mathrm{g}}\right\|=\infty .
$$


In the previous section we investigated the moments of $\phi(f)$ of positive integer order. By using Lemma 4.8 also the moments of fractional order can be derived. Define $|\phi(f)|^{s}=\left(\phi(f)^{2}\right)^{s / 2}$ for $0 \leq s \leq 2$, and let $\lambda$ be the Lévy measure on $\mathbb{R} \backslash\{0\}$ such that $\int_{0}^{\infty}\left(1-e^{-y u}\right) \lambda(d y)=u^{s / 2}$ for $u>0$, i.e.,

$$
\lambda(d y)=\frac{s}{2 \Gamma(1-s / 2)} y^{-1-\frac{s}{2}} 1_{(0, \infty)}(y) d y,
$$

corresponding to the $s / 2$-subordinator. Let $\Lambda_{\alpha}=\left\||\phi(f)|^{s / 2} \varphi_{\mathrm{g}}\right\|^{2}$.

Corollary 4.11 Suppose that $\hat{h} / \omega \in L^{2}\left(\mathbb{R}^{d}\right)$ and $f \in L^{2}\left(\mathbb{R}^{d}\right)$ is a real-valued function. Then for $0<s<2$,

$$
\Lambda_{\alpha}=\mathbb{E}_{\mu_{\mathrm{g}}}\left[\int_{0}^{\infty}\left(1-\frac{1}{\sqrt{1+\beta\|f\|^{2}}} e^{-\frac{\beta K(f)^{2}}{1+\beta\|f\|^{2}}}\right) \lambda(d \beta)\right] .
$$

In particular, $\Lambda_{0} \leq \Lambda_{\alpha}$ follows.

Proof: Notice that

$$
\begin{aligned}
\left(1-\frac{1}{\sqrt{1+\beta\|f\|^{2}}} e^{-\frac{\beta K(f)^{2}}{1+\beta\|f\|^{2}}}\right) \beta^{-1-\frac{s}{2}} & \leq\left(1-\frac{1}{\sqrt{1+\beta\|f\|^{2}}} e^{-\frac{\beta \alpha^{2}\|f\|^{2}\|\hat{h} / \omega\|^{2} / 4}{1+\beta\|f\|^{2}}}\right) \beta^{-1-\frac{s}{2}} \\
& =\eta(\beta) .
\end{aligned}
$$

In a neighborhood of $\beta=0$ it holds that $\eta(\beta)=\beta^{-s / 2}+o(\beta)$ locally uniformly. Then $\eta(\beta)$ is integrable in this neighborhood, and since $\eta(\beta) \leq \operatorname{const} \beta^{1+s / 2}, \eta \in L^{1}([0, \infty))$ follows. Then (4.25) is immediate from Lemma 4.8 by using the Fubini theorem, and the inequality follows from

$$
\Lambda_{0}=\int_{0}^{\infty}\left(1-\frac{1}{\sqrt{1+\beta\|f\|^{2}}}\right) \lambda(d \beta) .
$$

Remark 4.12 A simple computation shows that if $U$ is a real-valued Gaussian random variable with mean $m$ and variance $\sigma^{2}$, then whenever $\theta<1 /\left(2 \sigma^{2}\right)$, we have

$$
\mathbb{E}_{G}\left[e^{\theta U^{2}}\right]=\frac{1}{\sqrt{1-2 \theta \sigma^{2}}} e^{\frac{m^{2} \theta}{1-2 \theta \sigma^{2}}},
$$

where the expectation is taken with respect to this Gaussian measure. A comparison with (4.17) implies that there exists thus a real-valued Gaussian random variable $U$ with mean $K(f)$ (or $-K(f))$ and variance $\|f\|^{2} / 2$ such that

$$
\left\|e^{\beta \phi(f)^{2}} \varphi_{\mathrm{g}}\right\|^{2}=\mathbb{E}_{G}\left[e^{\beta U^{2}}\right] .
$$

For some consequences see Section 4.3 below. 


\subsubsection{Exponential moments of the field operator}

Theorem 4.9 says that $\left\|e^{(\beta / 2) \phi(f)^{2}} \varphi_{\mathrm{g}}\right\|<\infty$. Using this fact we can obtain explicit formulae of the exponential moments $\left(\varphi_{\mathrm{g}}, e^{\beta \phi(f)} \varphi_{\mathrm{g}}\right)$ of the field.

Corollary 4.13 If $\hat{h} / \omega \in L^{2}\left(\mathbb{R}^{d}\right)$ and $f \in L^{2}\left(\mathbb{R}^{d}\right)$ is a real-valued function, then $\varphi_{\mathrm{g}} \in$ $D\left(e^{\beta \phi(f)}\right)$ and

$$
\begin{aligned}
& \left(\varphi_{\mathrm{g}}, e^{\beta \phi(f)} \varphi_{\mathrm{g}}\right)=\left(\varphi_{\mathrm{g}}, \cosh (\beta \phi(f)) \varphi_{\mathrm{g}}\right)=e^{\frac{\beta^{2}}{4}\|f\|^{2}} \mathbb{E}_{\mu_{\mathrm{g}}}\left[e^{\beta K(f)}\right], \\
& \left(\varphi_{\mathrm{g}}, \sigma e^{\beta \phi(f)} \varphi_{\mathrm{g}}\right)=\left(\varphi_{\mathrm{g}}, \sigma \sinh (\beta \phi(f)) \varphi_{\mathrm{g}}\right)=e^{\frac{\beta^{2}}{4}\|f\|^{2}} \mathbb{E}_{\mu_{\mathrm{g}}}\left[X_{0} e^{\beta K(f)}\right] .
\end{aligned}
$$

Proof: For simplicity we reset $\beta f$ to $f$. By using the generating function $e^{x y-\frac{1}{2} y^{2}}=$ $\sum_{n=0}^{\infty} h_{n}(x) \frac{y^{n}}{n !}$ of the Hermite polynomials, summation in (4.8) gives

$$
\lim _{M \rightarrow \infty}\left(\varphi_{\mathrm{g}}, \sum_{n=0}^{M} \frac{1}{n !} \phi(f)^{n} \varphi_{\mathrm{g}}\right)=e^{\frac{1}{4}\|f\|^{2}} \mathbb{E}_{\mu_{\mathrm{g}}}\left[e^{K(f)}\right] .
$$

We need to check that the left hand side converges to $\left(\varphi_{\mathrm{g}}, e^{\phi(f)} \varphi_{\mathrm{g}}\right)$. Notice that by the spin flip property (2) in Lemma 1.6, $\left(\varphi_{\mathrm{g}}, \phi(f)^{n} \varphi_{\mathrm{g}}\right)=0$ for odd $n$. Hence it suffices to show the convergence of $\left(\varphi_{\mathrm{g}}, \sum_{n=0}^{M} \frac{1}{(2 n) !} \phi(f)^{2 n} \varphi_{\mathrm{g}}\right)$ as $M \rightarrow \infty$. By Theorem 4.9 we have that $\left\|e^{\phi(f)^{2} /\left(4\|f\|^{2}\right)} \varphi_{\mathrm{g}}\right\|<\infty$. Let $E_{\lambda}$ be the spectral measure of $\phi(f)$ with respect to $\varphi_{\mathrm{g}}$. Then

$$
\sum_{n=0}^{M} \frac{1}{(2 n) !}\left(\varphi_{\mathrm{g}}, \phi(f)^{2 n} \varphi_{\mathrm{g}}\right)=\int_{\mathbb{R}} \sum_{n=0}^{M} \frac{1}{(2 n) !} \lambda^{2 n} e^{-\lambda^{2} /\left(4\|f\|^{2}\right)} e^{\lambda^{2} /\left(4\|f\|^{2}\right)} d E_{\lambda} .
$$

Since $e^{\lambda^{2} /\left(4\|f\|^{2}\right)}$ is integrable by Theorem 4.9, $\sum_{n=0}^{M} \frac{1}{(2 n) !} \lambda^{2 n} e^{-\lambda^{2} /\left(4\|f\|^{2}\right)}$ is monotonously increasing to $\cosh (\lambda) e^{-\lambda^{2} /\left(4\|f\|^{2}\right)}$ as $M \uparrow \infty$, which is a bounded function, hence the monotone convergence theorem yields that $\lim _{M \rightarrow \infty} \int_{\mathbb{R}} \sum_{n=0}^{M} \frac{1}{n !} \lambda^{n} d E_{\lambda}=\int_{\mathbb{R}} e^{\lambda} d E_{\lambda}<\infty$, which implies $\varphi_{\mathrm{g}} \in D\left(e^{\phi(f)}\right)$ and (4.26). Equality (4.27) is derived in a similar way.

\subsection{Van Hove representation}

In Remark 4.12 we pointed out that the expectation of the field operator $\phi(f)$ in the ground state $\varphi_{\mathrm{g}}$ can be realized as an expectation of a Gaussian random variable. Here we show that this allows another representation of the ground state.

The van Hove Hamiltonian is defined by the self-adjoint operator

$$
H_{\mathrm{vH}}(\hat{g})=H_{\mathrm{f}}+\phi_{\mathrm{b}}(\hat{g})
$$


in Fock space $\mathscr{F}$. Suppose that $\hat{g} / \omega \in L^{2}\left(\mathbb{R}^{d}\right)$ and define the conjugate momentum by

$$
\pi_{\mathrm{b}}(\hat{g})=\frac{i}{\sqrt{2}} \int\left(a^{\dagger}(k) \frac{\hat{g}(k)}{\omega(k)}-a(k) \frac{\hat{g}(-k)}{\omega(k)}\right) d k .
$$

Then

$$
e^{i \pi_{\mathrm{b}}(\hat{g})} H_{\mathrm{vH}}(\hat{g}) e^{-i \pi_{\mathrm{b}}(\hat{g})}=H_{\mathrm{f}}-\frac{1}{2}\|\hat{g} / \omega\|^{2}
$$

and the ground state of $H_{\mathrm{vH}}(\hat{g})$ is given by

$$
\varphi_{\mathrm{vH}}(\hat{g})=e^{-i \pi_{\mathrm{b}}(\hat{g})} \Omega_{\mathrm{b}} .
$$

On the other hand, clearly the spin-boson Hamiltonian $H$ with $\varepsilon=0$ is the direct sum of van Hove Hamiltonians since

$$
H=\left[\begin{array}{cc}
H_{\mathrm{f}}+\alpha \phi_{\mathrm{b}}(\hat{h}) & 0 \\
0 & H_{\mathrm{f}}-\alpha \phi_{\mathrm{b}}(\hat{h})
\end{array}\right]
$$

(see Section 2) and $H_{\mathrm{f}} \pm \alpha \phi_{\mathrm{b}}(\hat{h})$ are equivalent. Therefore the ground state of $H$ with $\varepsilon=0$ can be realized as $\varphi_{\mathrm{g}}=\left[\begin{array}{c}\varphi_{\mathrm{vH}}(\alpha \hat{h}) \\ \varphi_{\mathrm{vH}}(-\alpha \hat{h})\end{array}\right]$. Thus in this case

$$
\left(\varphi_{\mathrm{g}}, e^{i \beta \phi(f)} \varphi_{\mathrm{g}}\right)_{\mathscr{H}}=\left(\varphi_{\mathrm{vH}}(\alpha \hat{h}), e^{i \beta \phi_{\mathrm{b}}(\hat{f})} \varphi_{\mathrm{vH}}(\alpha \hat{h})\right)_{\mathscr{F}}
$$

and the right hand side above equals

$$
\left(\Omega_{\mathrm{b}}, e^{i \beta\left(\phi_{\mathrm{b}}(\hat{f})+\alpha(\hat{h} / \omega, \hat{f})\right)} \Omega_{\mathrm{b}}\right)_{\mathscr{F}}=e^{-\beta^{2}\|\hat{f}\|^{2} / 4+i \beta \alpha(\hat{h} / \omega, \hat{f})} .
$$

When $\varepsilon \neq 0$ we can derive similar but non-trivial representations. Define the random boson field operator

$$
\Psi(\hat{f})=\phi_{\mathrm{b}}(\hat{f})+K(f)
$$

on $\mathscr{F}$, where $K(f)$ is the random variable on $\mathscr{X}$ defined by (4.7). Then we see that

$$
\begin{aligned}
& \left(\Omega_{\mathrm{b}}, \Psi(\hat{f}) \Omega_{\mathrm{b}}\right)=K(f), \\
& \left(\Omega_{\mathrm{b}}, \Psi(\hat{f})^{2} \Omega_{\mathrm{b}}\right)-\left(\Omega_{\mathrm{b}}, \Psi(\hat{f}) \Omega_{\mathrm{b}}\right)^{2}=\|\hat{f}\|^{2} / 2, \\
& \left(\Omega_{\mathrm{b}}, e^{i \beta \Psi(\hat{f})} \Omega_{\mathrm{b}}\right)=e^{-\beta^{2}\|f\|^{2} / 4+i \beta K(f)} .
\end{aligned}
$$

Let

$$
\chi=\frac{\alpha}{2} \omega(k) \hat{h}(k) \int_{-\infty}^{\infty} e^{-|s| \omega(k)} X_{\varepsilon s} d s
$$

Note that $\chi \in L^{2}\left(\mathbb{R}^{d}\right)$

$$
K(f)=(\chi, \hat{f}),
$$

moreover, $\chi / \omega \in L^{2}\left(\mathbb{R}^{d}\right)$, whenever $\hat{h} / \omega \in L^{2}\left(\mathbb{R}^{d}\right)$, and $\chi=\alpha \hat{h}$ for $\varepsilon=0$. We define the random van Hove Hamiltonian by $H_{\mathrm{vH}}(\chi)$. 
Theorem 4.14 If $\hat{h} / \omega \in L^{2}\left(\mathbb{R}^{d}\right)$, then

$$
\left(\varphi_{\mathrm{g}}, e^{i \beta \phi(f)} \varphi_{\mathrm{g}}\right)=\mathbb{E}_{\mu_{\mathrm{g}}}\left[\left(\Omega_{\mathrm{b}}, e^{i \beta \Psi(\hat{f})} \Omega_{\mathrm{b}}\right)\right]=\mathbb{E}_{\mu_{\mathrm{g}}}\left[\left(\varphi_{\mathrm{vH}}(\chi), e^{i \beta \phi_{\mathrm{b}}(\hat{f})} \varphi_{\mathrm{vH}}(\chi)\right)\right] .
$$

Proof: The first equality can be directly derived from Theorem 4.4. The second equality follows from $e^{i \pi_{\mathrm{b}}(\chi)} \Psi(\hat{f}) e^{-i \pi_{\mathrm{b}}(\chi)}=\phi_{\mathrm{b}}(\hat{f})$.

Corollary 4.15 Suppose $\hat{h} / \omega \in L^{2}\left(\mathbb{R}^{d}\right)$ and $F \in \mathscr{S}(\mathbb{R})$. Then we have

$$
\begin{aligned}
& \left(\varphi_{\mathrm{g}}, F(\phi(f)) \varphi_{\mathrm{g}}\right)=\mathbb{E}_{\mu_{\mathrm{g}}}\left[\left(\Omega_{\mathrm{b}}, F(\Psi(\hat{f})) \Omega_{\mathrm{b}}\right)\right]=\mathbb{E}_{\mu_{\mathrm{g}}}\left[\left(\varphi_{\mathrm{vH}}(\chi), F(\phi(\hat{f})) \varphi_{\mathrm{vH}}(\chi)\right)\right], \\
& \left\|e^{\beta \phi(f)^{2} / 2} \varphi_{\mathrm{g}}\right\|^{2}=\mathbb{E}_{\mu_{\mathrm{g}}}\left[\left\|e^{\beta \Psi(\hat{f})^{2} / 2} \Omega_{\mathrm{b}}\right\|^{2}\right]=\mathbb{E}_{\mu_{\mathrm{g}}}\left[\left\|e^{\beta \phi_{\mathrm{b}}(\hat{f})^{2} / 2} \varphi_{\mathrm{vH}}(\chi)\right\|^{2}\right] .
\end{aligned}
$$

Proof: This is proven from Corollary 4.6 and Theorem 4.9.

\subsection{Expectations of second quantized operators}

\subsubsection{General results}

In this section we consider expectations of the form $\left(\varphi_{\mathrm{g}}, e^{-\beta d \Gamma(\rho(-i \nabla))} \varphi_{\mathrm{g}}\right)$, where $\rho$ is a real-valued multiplication operator given by the function $\rho$. An important example is $\rho=\mathbb{1}$ giving the boson number operator $N=d \Gamma(\mathbb{1})$.

In a similar way to [GHPS09, Section 3.2] we obtain the expression

$$
\frac{\left(\Phi_{T}, \xi(\sigma) e^{-\beta d \Gamma(\rho(-i \nabla))} \Phi_{T}\right)}{\left\|\Phi_{T}\right\|^{2}}=\mathbb{E}_{\mu_{T}^{\varepsilon}}\left[\xi\left(X_{0}\right) e^{-\alpha^{2} \int_{-T}^{0} d t \int_{0}^{T} W^{\rho, \beta}\left(X_{\varepsilon t}, X_{\varepsilon s}, t-s\right) d s}\right],
$$

where

$$
W^{\rho, \beta}(x, y, T)=\frac{x y}{2} \int_{\mathbb{R}^{d}}|\hat{h}(k)|^{2} e^{-|T| \omega(k)}\left(1-e^{-\beta \rho(k)}\right) d k .
$$

Denote

$$
W_{\infty}^{\rho, \beta}=\int_{-\infty}^{0} d t \int_{0}^{\infty} W^{\rho, \beta}\left(X_{\varepsilon t}, X_{\varepsilon s}, t-s\right) d s
$$

Notice that $\left|W_{\infty}^{\rho, \beta}\right| \leq\|\hat{h} / \omega\|^{2} / 2<\infty$, uniformly in the paths in $\mathscr{X}$.

Theorem 4.16 Suppose that $\hat{h} / \omega \in L^{2}\left(\mathbb{R}^{d}\right)$ and $\xi: \mathbb{Z}_{2} \rightarrow \mathbb{C}$ is a bounded function. Then

$$
\left(\varphi_{\mathrm{g}}, \xi(\sigma) e^{-\beta d \Gamma(\rho(-i \nabla))} \varphi_{\mathrm{g}}\right)=\mathbb{E}_{\mu_{\mathrm{g}}}\left[\xi\left(X_{0}\right) e^{-\alpha^{2} W_{\infty}^{\rho, \beta}}\right], \quad \beta>0
$$


Proof: This is shown by using Theorem 3.8 and telescoping. For a shorthand we write $W_{T}^{\rho, \beta}=\int_{-T}^{0} d s \int_{0}^{T} W^{\rho, \beta}\left(X_{\varepsilon t}, X_{\varepsilon s}, t-s\right) d t$. Note that for every $\delta>0$ there is $S_{\delta}$ such that $\left|W_{T}^{\rho, \beta}-W_{\infty}^{\rho, \beta}\right| \leq \delta$ for all $T>S_{\delta}$, uniformly in the paths, and write

$$
\begin{aligned}
& \mathbb{E}_{\mu_{T}^{\varepsilon}}\left[\xi\left(X_{0}\right) e^{-\alpha^{2} W_{T}^{\rho, \beta}}\right]-\mathbb{E}_{\mu_{\mathrm{g}}}\left[\xi\left(X_{0}\right) e^{-\alpha^{2} W_{\infty}^{\rho, \beta}}\right] \\
& =\mathbb{E}_{\mu_{T}^{\varepsilon}}\left[\xi\left(X_{0}\right) e^{-\alpha^{2} W_{T}^{\rho, \beta}}\right]-\mathbb{E}_{\mu_{T}^{\varepsilon}}\left[\xi\left(X_{0}\right) e^{-\alpha^{2} W_{\infty}^{\rho, \beta}}\right] \\
& \quad+\mathbb{E}_{\mu_{T}^{\varepsilon}}\left[\xi\left(X_{0}\right) e^{-\alpha^{2} W_{\infty}^{\rho, \beta}}\right]-\mathbb{E}_{\mu_{\mathrm{g}}}\left[\xi\left(X_{0}\right) e^{-\alpha^{2} W_{\infty}^{\rho, \beta}}\right] .
\end{aligned}
$$

We have

$$
\left|\mathbb{E}_{\mu_{T}^{\varepsilon}}\left[\xi\left(X_{0}\right) e^{-\alpha^{2} W_{T}^{\rho, \beta}}\right]-\mathbb{E}_{\mu_{T}^{\varepsilon}}\left[\xi\left(X_{0}\right) e^{-\alpha^{2} W_{\infty}^{\rho, \beta}}\right]\right| \leq C \delta
$$

with a constant $C$. The second term can be evaluated as

$$
\begin{aligned}
\left|\mathbb{E}_{\mu_{T}^{\varepsilon}}\left[\xi\left(X_{0}\right) e^{-\alpha^{2} W_{\infty}^{\rho, \beta}}\right]-\mathbb{E}_{\mu_{\mathrm{g}}}\left[\xi\left(X_{0}\right) e^{-\alpha^{2} W_{\infty}^{\rho, \beta}}\right]\right| \\
\leq\left|\mathbb{E}_{\mu_{T}^{\varepsilon}}\left[\xi\left(X_{0}\right) e^{-\alpha^{2} W_{\infty}^{\rho, \beta}}\right]-\mathbb{E}_{\mu_{T}^{\varepsilon}}\left[\xi\left(X_{0}\right) e^{-\alpha^{2} W_{S_{\delta}}^{\rho, \beta}}\right]\right| \\
+\left|\mathbb{E}_{\mu_{T}^{\varepsilon}}\left[\xi\left(X_{0}\right) e^{-\alpha^{2} W_{S_{\delta}}^{\rho, \beta}}\right]-\mathbb{E}_{\mu_{\mathrm{g}}}\left[\xi\left(X_{0}\right) e^{-\alpha^{2} W_{S_{\delta}}^{\rho, \beta}}\right]\right| \\
+\left|\mathbb{E}_{\mu_{\mathrm{g}}}\left[\xi\left(X_{0}\right) e^{-\alpha^{2} W_{S_{\delta}}^{\rho, \beta}}\right]-\mathbb{E}_{\mu_{\mathrm{g}}}\left[\xi\left(X_{0}\right) e^{-\alpha^{2} W_{\infty}^{\rho, \beta}}\right]\right| .
\end{aligned}
$$

For (4.46) and (4.48) we have again the same upper bound as in (4.45), and (4.47) goes to zero as $T \rightarrow \infty$ by Theorem 3.8.

\subsubsection{Super-exponential decay of the boson number}

In this section we discuss the expectation of $e^{-\beta N}$, which can be obtained by a minor modification of Theorem 4.16.

Corollary 4.17 Suppose that $\hat{h} / \omega \in L^{2}\left(\mathbb{R}^{d}\right)$ and $\xi: \mathbb{Z}_{2} \rightarrow \mathbb{C}$ is a bounded function. Then

$$
\left(\varphi_{\mathrm{g}}, \xi(\sigma) e^{-\beta N} \varphi_{\mathrm{g}}\right)=\mathbb{E}_{\mu_{\mathrm{g}}}\left[\xi\left(X_{0}\right) e^{-\alpha^{2}\left(1-e^{-\beta}\right) W_{\infty}}\right]
$$

where

$$
W_{\infty}=\int_{-\infty}^{0} d t \int_{0}^{\infty} W\left(X_{\varepsilon t}, X_{\varepsilon s}, t-s\right) d s
$$

Proof: By replacing $\rho$ by $\mathbb{1}$ in Theorem 4.16 , the claim readily follows.

The following result says that the distribution of the number of bosons in the ground state has a super-exponentially short tail. 
Corollary 4.18 If $\hat{h} / \omega \in L^{2}\left(\mathbb{R}^{d}\right)$, then $\varphi_{\mathrm{g}} \in D\left(e^{\beta N}\right)$ for all $\beta \in \mathbb{C}$ and

$$
\left(\varphi_{\mathrm{g}}, e^{\beta N} \varphi_{\mathrm{g}}\right)=\mathbb{E}_{\mu_{\mathrm{g}}}\left[e^{-\alpha^{2}\left(1-e^{\beta}\right) W_{\infty}}\right]
$$

follows. In particular, $\varphi_{\mathrm{g}} \in D\left(e^{+\beta N}\right)$ for all $\beta>0$.

Proof: The proof is similar to that of Theorem 4.9 and [Hir04, Theorem 10.12], and is left to the reader.

Corollary 4.19 Suppose $\hat{h} / \omega \in L^{2}\left(\mathbb{R}^{d}\right)$. Then

$$
\begin{aligned}
& \left(\varphi_{\mathrm{g}},(-1)^{N} \varphi_{\mathrm{g}}\right)=\mathbb{E}_{\mu_{\mathrm{g}}}\left[e^{-2 \alpha^{2} W_{\infty}}\right], \\
& \left(\varphi_{\mathrm{g}}, \xi(\sigma)(-1)^{N} \varphi_{\mathrm{g}}\right)=\mathbb{E}_{\mu_{\mathrm{g}}}\left[\xi\left(X_{0}\right) e^{-2 \alpha^{2} W_{\infty}}\right] .
\end{aligned}
$$

In particular, it follows that

$$
\begin{aligned}
& \left(\varphi_{\mathrm{g}},(-1)^{N} \varphi_{\mathrm{g}}\right)=\mathbb{E}_{\mu_{\mathrm{g}}}\left[e^{-2 \alpha^{2} W_{\infty}}\right] \geq e^{-\alpha^{2}\|\hat{h} / \omega\|^{2}}>0, \\
& \left(\varphi_{\mathrm{g}}, \sigma(-1)^{N} \varphi_{\mathrm{g}}\right)=\mathbb{E}_{\mu_{\mathrm{g}}}\left[X_{0} e^{-2 \alpha^{2} W_{\infty}}\right]=-1<0 .
\end{aligned}
$$

Proof: Equality (4.52) is derived from (4.49) with $\xi(\sigma)=1$ and $\beta=-i \pi$, and (4.53) with $\xi(\sigma)=\sigma$. Equality (4.54) follows from the estimate of the right hand side of (4.53). Noticing that $\varphi_{\mathrm{SB}} \in \mathscr{H}_{-}$, we obtain $P \varphi_{\mathrm{SB}}=\sigma_{x}(-1)^{N} \varphi_{\mathrm{SB}}=-\varphi_{\mathrm{SB}}$. In particular, this gives

$$
\mathbb{E}_{\mu_{\mathrm{g}}}\left[X_{0} e^{-2 \alpha^{2} W_{\infty}}\right]=\left(\varphi_{\mathrm{g}}, \sigma(-1)^{N} \varphi_{\mathrm{g}}\right)=\left(\varphi_{\mathrm{SB}}, P \varphi_{\mathrm{SB}}\right)=-1
$$

\subsubsection{Moments of the boson number operator}

We can derive the expectation of $N^{m}, m=1,2, \ldots$, with respect to the ground state $\varphi_{\mathrm{g}}$ by using Corollary 4.17 .

Corollary 4.20 Suppose that $\hat{h} / \omega \in L^{2}\left(\mathbb{R}^{d}\right)$. Then

$$
\left(\varphi_{\mathrm{g}}, N^{m} \varphi_{\mathrm{g}}\right)=\sum_{r=1}^{m} a_{r}(m) \alpha^{2 r} \mathbb{E}_{\mu_{\mathrm{g}}}\left[W_{\infty}^{r}\right], \quad m=1,2,3, \ldots
$$

where $a_{r}(m)=\frac{(-1)^{r}}{r !} \sum_{s=1}^{r}(-1)^{s}\left(\begin{array}{l}r \\ s\end{array}\right) s^{m}$ are the Stirling numbers of the second kind.

Proof: It can be checked that

$$
\frac{d^{m}}{d \beta^{m}} e^{-C\left(1-e^{-\beta}\right)}=(-1)^{m} \sum_{r=1}^{m} a_{r}(m) e^{-r \beta}(-C)^{r} e^{-a\left(1-e^{-\beta}\right)} .
$$

Then the corollary follows from $\left(\varphi_{\mathrm{g}}, N^{m} \varphi_{\mathrm{g}}\right)=(-1)^{m} \frac{d^{m}}{d \beta^{m}}\left(\varphi_{\mathrm{g}}, e^{-\beta N} \varphi_{\mathrm{g}}\right)\left\lceil_{\beta=0}\right.$ and Corollary 4.17 . 


\subsection{A relation between the expectations of $\sigma \phi(f)$ and $N$}

By the results obtained in the previous subsections we can derive an inequality connecting the expectations $\left(\varphi_{\mathrm{g}}, \sigma \phi(f) \varphi_{\mathrm{g}}\right)$ and $\left(\varphi_{\mathrm{g}}, N \varphi_{\mathrm{g}}\right)$.

Corollary 4.21 Suppose that $\hat{h} / \omega \in L^{2}\left(\mathbb{R}^{d}\right), f \in L^{2}\left(\mathbb{R}^{d}\right)$ is real-valued, and $\xi: \mathbb{Z}_{2} \rightarrow \mathbb{C}$ a bounded function. Then

$$
\left(\varphi_{\mathrm{g}}, \xi(\sigma) \phi(f) \varphi_{\mathrm{g}}\right)=\alpha \int_{\mathbb{R}^{d}}\left(\xi(\sigma) \varphi_{\mathrm{g}},(H-E+\omega(k))^{-1} \sigma \varphi_{\mathrm{g}}\right) \overline{\hat{h}}(k) \hat{f}(k) d k
$$

In particular,

$$
\left(\varphi_{\mathrm{g}}, \sigma \phi(h) \varphi_{\mathrm{g}}\right)=\alpha \int_{\mathbb{R}^{d}}\left\|(H-E+\omega(k))^{-1 / 2} \sigma \varphi_{\mathrm{g}}\right\|^{2}|\hat{h}(k)|^{2} d k .
$$

Proof: By Theorem 4.4 we have

$$
\left(\varphi_{\mathrm{g}}, \xi(\sigma) \phi(f) \varphi_{\mathrm{g}}\right)=\mathbb{E}_{\mu_{\mathrm{g}}}\left[\xi\left(X_{0}\right) K(f)\right]=\frac{\alpha}{2} \int_{-\infty}^{\infty} d r\left(e^{-|r| \omega} \hat{h}, \hat{f}\right) \mathbb{E}_{\mu_{\mathrm{g}}}\left[\xi\left(X_{0}\right) X_{\varepsilon r}\right] .
$$

By Corollary 4.2 we also see that this furthermore is

$$
\begin{aligned}
& =\frac{\alpha}{2} \int_{-\infty}^{\infty} d r \int d k\left(\xi(\sigma) \varphi_{\mathrm{g}}, e^{-|r|(H-E+\omega(k))} \sigma \varphi_{\mathrm{g}}\right) \overline{\hat{h}(k)} \hat{f}(k) \\
& =\alpha \int_{\mathbb{R}^{d}}\left(\xi(\sigma) \varphi_{\mathrm{g}},(H-E+\omega(k))^{-1} \sigma \varphi_{\mathrm{g}}\right) \overline{\hat{h}(k)} \hat{f}(k) d k
\end{aligned}
$$

Then the corollary follows.

A standard inequality, see e.g. [LHB11, Proposition 5.1], says that

$$
\left(\Phi,(\sigma \phi(f))^{2} \Phi\right) \leq \frac{\|f\|^{2}}{2}(\Phi,(N+\mathbb{1}) \Phi) .
$$

From Corollary 4.21 we obtain the following inequality.

Corollary 4.22 Let $\hat{h} / \omega \in L^{2}\left(\mathbb{R}^{d}\right)$. Then

$$
\left(\varphi_{\mathrm{g}}, N \varphi_{\mathrm{g}}\right) \leq \frac{\alpha}{2}\left(\varphi_{\mathrm{g}}, \sigma \phi\left(\omega(\mathrm{D})^{-1} h\right) \varphi_{\mathrm{g}}\right) \leq \frac{\alpha^{2}}{2}\|\hat{h} / \omega\|^{2}, \quad \mathrm{D}=-i \nabla .
$$

Proof: By (4.56) we have

$$
\left(\varphi_{\mathrm{g}}, N \varphi_{\mathrm{g}}\right)=\frac{\alpha^{2}}{2} \int_{\mathbb{R}^{d}}|\hat{h}(k)|^{2} \int_{-\infty}^{0} d t \int_{0}^{\infty} d s e^{-|t-s| \omega(k)} \mathbb{E}_{\mu_{\mathrm{g}}}\left[X_{\varepsilon t} X_{\varepsilon s}\right] .
$$


Since $\mathbb{E}_{\mu_{\mathrm{g}}}\left[X_{\varepsilon s} X_{\varepsilon t}\right]=\left(\sigma \varphi_{\mathrm{g}}, e^{-|t-s|(H-E)} \sigma \varphi_{\mathrm{g}}\right)$, by Corollary 4.3 we see that

$$
\left(\varphi_{\mathrm{g}}, N \varphi_{\mathrm{g}}\right)=\frac{\alpha^{2}}{2} \int_{\mathbb{R}^{d}}|\hat{h}(k)|^{2}\left\|(H-E+\omega(k))^{-1} \sigma \varphi_{\mathrm{g}}\right\|^{2} d k .
$$

The first inequality is derived from (4.60) and (4.58). The second inequality follows through (4.58).

Since $\varphi_{\mathrm{g}} \in D(N)$, we have that $f \mapsto\left(\varphi_{\mathrm{g}}, \sigma \phi(f) \varphi_{\mathrm{g}}\right)$ is linear and the bound $\left|\left(\varphi_{\mathrm{g}}, \sigma \phi(f) \varphi_{\mathrm{g}}\right)\right| \leq C\|f\|$ with a constant $C$ follows. By the Riesz representation theorem there exists $G \in L^{2}\left(\mathbb{R}^{d}\right)$ such that $\left(\varphi_{\mathrm{g}}, \sigma \phi(f) \varphi_{\mathrm{g}}\right)=(\hat{G}, \hat{f})_{L^{2}\left(\mathbb{R}^{d}\right)}$.

Corollary 4.23 If $\hat{h} / \omega \in L^{2}\left(\mathbb{R}^{d}\right)$, then

$$
\hat{G}(k)=\frac{\alpha}{2}\left(\sigma \varphi_{\mathrm{g}},(H-E+\omega(k))^{-1} \sigma \varphi_{\mathrm{g}}\right) \hat{h}(k) .
$$

Proof: This is obtained directly from Corollary 4.21 .

\subsection{Comparison with the Nelson model}

From (4.61) we see that, formally, $G(\cdot)=\left(\varphi_{\mathrm{g}}, \sigma \phi(\cdot) \varphi_{\mathrm{g}}\right)$. Recall that the Nelson model is defined by a linear coupling between a particle described by the Schrödinger operator $H_{\mathrm{p}}=-\Delta+V$ and a boson field described by $H_{\mathrm{f}}$. The coupling term is given by

$$
\phi_{\mathrm{b}}(x)=\frac{1}{\sqrt{2}} \int\left(a^{\dagger}(k) e^{-i k x} \hat{h}(-k)+a(k) e^{-i k x} \hat{h}(k)\right) d k
$$

so that the Nelson Hamiltonian is defined by

$$
H_{\mathrm{N}}=H_{\mathrm{p}}+H_{\mathrm{f}}+\phi_{\mathrm{b}}(x)
$$

For this model a similar kernel to (4.61) is obtained, see [LHB11, eq. (6.5.60)]. Using this kernel we can show [LHB11, Sect. 6.5] that

$$
a\|\hat{h} / \omega\|^{2}-b \leq\left(\varphi_{\mathrm{g}}^{\mathrm{N}}, N \varphi_{\mathrm{g}}^{\mathrm{N}}\right) \leq c\|\hat{h} / \omega\|^{2},
$$

where $\varphi_{\mathrm{g}}^{\mathrm{N}}$ is the ground state of $H_{\mathrm{N}}$, and $a, c \geq 0$ and $b \in \mathbb{R}$ are suitable constants. Thus $\left(\varphi_{\mathrm{g}}^{\mathrm{N}}, N \varphi_{\mathrm{g}}^{\mathrm{N}}\right) \rightarrow \infty$ as $\|\hat{h} / \omega\| \rightarrow \infty$, and it follows that $H_{\mathrm{N}}$ has no ground state whenever $\hat{h} / \omega \notin L^{2}\left(\mathbb{R}^{d}\right)$. The key point is that $\left(\varphi_{\mathrm{g}}^{\mathrm{N}}, e^{i(\cdot, x)} \varphi_{\mathrm{g}}^{\mathrm{N}}\right) \neq 0$; for some discussions see [AHH99]. While in (4.59) we show the upper bound $\left(\varphi_{\mathrm{g}}, N \varphi_{\mathrm{g}}\right) \leq \frac{\alpha^{2}}{2}\|\hat{h} / \omega\|^{2}$, we have no lower bound like in (4.64) due to the fact that $\left(\varphi_{\mathrm{g}}, \sigma \varphi_{\mathrm{g}}\right)=0$. Although there 
is no mathematical proof known, the physics literature supports the conjecture that whenever a ground state exists, the boson number expectation $\left(\varphi_{\mathrm{g}}^{\mathrm{N}}, N \varphi_{\mathrm{g}}^{\mathrm{N}}\right)$ is finite. Thus $\hat{h} / \omega \notin L^{2}\left(\mathbb{R}^{d}\right)$ would then mean absence of a ground state. For the spin boson model it can be conjectured for similar physical reasons that the same mechanism applies and $\hat{h} / \omega \notin L^{2}\left(\mathbb{R}^{d}\right)$ implies absence of a ground state. Then some interesting open questions are if it is possible to prove $\left(\varphi_{\mathrm{g}}, N \varphi_{\mathrm{g}}\right) \rightarrow \infty$ as $\|\hat{h} / \omega\| \rightarrow \infty$ and, secondly, if a ground state of $H$ exists when $\hat{h} / \omega \notin L^{2}\left(\mathbb{R}^{d}\right)$. These questions will be considered elsewhere.

Acknowledgments: This work was financially supported by Grant-in-Aid for Science Research (B) 23340032 from JSPS. JL thanks IHES, Bures-sur-Yvette, for a visiting fellowship.

\section{References}

[Abd12] A. Abdesselam, The ground state energy of the massless spin-boson model, Ann. Henri Poincaré 12 (2011), 1321-1347.

[AMRZ] N. Angelescu, R. A. Minlos, J. Ruiz and V. Zagrebnov, Lower spectral branches of spin-boson model, J. Math. Phys. 49 (2008), 102105.

[AH97] A. Arai and M. Hirokawa, On the existence and uniqueness of ground states of generalized spin-boson model, J. Funct. Anal. 151 (1997), 455-503.

[AHH99] A. Arai, M. Hirokawa and F. Hiroshima, On the absence of eigenvectors of Hamiltonians in a class of massless quantum field models without infrared cutoff, J. Funct. Anal. 168 (1999), 470-497.

[BS98] A. Boutet de Monvel and J. Sahbani, On the spectral properties of the spin-boson Hamiltonians, Lett. Math. Phys. 44 (1998), 23-33.

[BH09] V. Betz and F. Hiroshima, Gibbs measures with double stochastic integrals on path space, Infinite Dimensional Analysis, Quantum Probability and Related Topics 12 (2009), 135-152.

[BHLMS02] V. Betz, F. Hiroshima, J. Lörinczi, R.A. Minlos and H. Spohn, Ground state properties of the Nelson Hamiltonian: a Gibbs measure-based approach, Rev. Math. Phys. 14 (2002), 173-198.

[FN88] M. Fannes and B. Nachtergaele, Translating the spin-boson model into a classical system, $J$. Math. Phys. 29 (1988), 2288-2293.

[Ger96] C. Gérard, Asymptotic completeness for the spin-boson model with a particle number cut, Rev. Math. Phys. 8 (1996), 549-589.

[Ger00] C. Gérard, On the existence of ground states for massless Pauli-Fierz Hamiltonians, Ann. Henri Poincaré 1 (2000), 443-459, and A remark on the paper: "On the existence of ground states for Hamiltonians", mp-arc 06-146 (2006).

[GHPS09] C. Gérard, F. Hiroshima, A. Panati and A. Suzuki, Infrared divergence of a scalar quantum field model on a pseudo Riemannian manifold, Interdisciplinary Information Science 15 (2009), 399-421. 
[GHPS12] C. Gérard, F. Hiroshima, A. Panati and A. Suzuki, Absence of ground state of the Nelson model with variable coefficients, J. Funct. Anal. 262 (2012), 273-299.

[Gro73] L. Gross, The relativistic polaron without cutoffs, Commun. Math. Phys. 31 (1973), 25-73.

[GL09] M. Gubinelli and J. Lőrinczi, Gibbs measures on Brownian currents, Commun. Pure Appl. Math. 62 (2009), 1-56.

[GHL12] M. Gubinelli, F. Hiroshima and J. Lörinczi, Ultraviolet renormalization of the Nelson model through functional integration, arXiv:1304.6662, 2013 (submitted for publication).

[HH10] D. Hasler and I. Herbst, Ground states in the spin boson model, arXiv:1003.5923v2 [math-ph], 2010.

[Hir99] M. Hirokawa, An expression of the ground state energy of the Spin-Boson model, J. Funct. Anal. 162 (1999), 178-218.

[Hir01] M. Hirokawa, Remarks on the ground state energy of the spin-boson model. An application of the Wigner-Weisskopf model, Rev. Math. Phys. 13 (2001), 221-251.

[Hir02] M. Hirokawa, Ground state transition for two-level system coupled with Bose field, Phys. Lett. A 294 (2002), 13-18.

[Hir06] M. Hirokawa, Infrared catastrophe for Nelson's model-non-existence of ground state and softboson divergence, Publ. Res. Inst. Math. Sci. 42 (2006), 897-922.

[HH12] M. Hirokawa and F. Hiroshima, Absence of energy level crossing for the ground state energy of the Rabi model, to appear in Commun. Stoch. Anal.

[Hir00] F. Hiroshima, Ground states of a model in nonrelativistic quantum electrodynamics II, $J$. Math. Phys. 41 (2000), 661-674.

[Hir04] F. Hiroshima, Analysis of ground states of atoms interacting with a quantized radiation field, Topics in the Theory of Schrödinger operators, H. Araki and H. Ezawa (eds.), World Scientific, $2004,145-272$.

[Hir12] F. Hiroshima, Functional integral approach to semi-relativistic Pauli-Fierz models, to appear in Adv. Math.

[HIL12a] F. Hiroshima, T. Ichinose, J. Lörinczi: Path integral representation for Schrödinger operators with Bernstein functions of the Laplacian, Rev. Math. Phys. 24 (2012), 1250013 (40 pages).

[HIL12b] F. Hiroshima, T. Ichinose and J. Lőrinczi, Probabilistic representation and fall-off of bound states of relativistic Schrödinger operators with spin 1/2, Publ. Res. Inst. Math. Sci. 49, 189-214, 2013.

[HL08] F. Hiroshima and J. Lörinczi, Functional integral representation of the Pauli-Fierz Hamiltonian with spin 1/2, J. Funct. Anal. 254 (2008), 2127-2185.

[HS95] M. Hübner and H. Spohn, Spectral properties of the spin-boson Hamiltonian, Ann. Inst. Henri Poincaré 62 (1995), 289-323.

[KL12a] K. Kaleta and J. Lőrinczi, Fractional $P(\phi)_{1}$-processes and Gibbs measures, Stoch. Proc. Appl. 122 (2012), 3580-3617.

[KL12b] K. Kaleta and J. Lőrinczi, Pointwise eigenfunction estimates and intrinsic ultracontractivitytype properties of Feynman-Kac semigroups for a class of Lévy processes, to appear in Ann. Probab. 
[LCDGZ87] A.J. Leggett, S. Chakravarty, A.T. Dorsey, M.P. Fisher, A. Garg, and W. Zwerger, Dynamics of a dissipative two-state system, Rev. Mod. Phys 59 (1987), 1-85, Erratum: Rev. Mod. Phys. 67 (1995), 725.

[L02] J. Lörinczi, Gibbs measures on Brownian paths, in In and Out of Equilibrium: Physics with a Probability Flavour, V. Sidoravicius, ed., Birkhäuser, pp. 349-362, 2002

[LHB11] J. Lőrinczi, F. Hiroshima and V. Betz, Feynman-Kac-Type Theorems and Gibbs Measures on Path Space, de Gruyter Studies in Mathematics 34, 2011.

[LM12] J. Lörinczi and J. Małecki, Spectral properties of the massless relativistic harmonic oscillator, J. Diff. Equations 253 (2012), 2846-2871.

[LM01] J. Lörinczi and R.A. Minlos, Gibbs measures for Brownian paths under the effect of an external and a small pair potential, J. Stat. Phys. 105 (2001), 605-647.

[LMS02] J. Lörinczi, R.A. Minlos and H. Spohn, The infrared behavior in Nelson's model of a quantum particle coupled to a massless scalar field, Ann. Henri Poincaré 3 (2002), 1-28.

[Spo89] H. Spohn, Ground state(s) of the spin-boson Hamiltonian, Commun. Math. Phys. 123 (1989), $277-304$.

[SD85] H. Spohn and R. Dümcke, Quantum tunneling with dissipation and the Ising model over $\mathbb{R}$, J. Stat. Phys. 41 (1985), 389-423.

[SSW90] H. Spohn, R. Stück and W. Wresziński, Localisation for the spin J-boson Hamiltonian, Ann. Inst. Henri Poincaré 53 (1990), 225-244. 